\title{
FEASIBILITY OF COMP USAGE IN BASE STATION SWITCHING OFF DURING OFF-PEAK PERIODS
}

by

Abid Jan

Master of Business Administration, University of Peshawar, 2012

Master of Science in Electrical Engineering, NWFP UET Peshawar, 2005

Bachelor of Science in Electrical Engineering, NWFP UET Peshawar, 1999

\author{
A thesis \\ presented to Ryerson University \\ in partial fulfillment of the \\ requirements for the degree of \\ Master of Applied Science \\ in the Program of \\ Electrical and Computer Engineering
}

Toronto, Ontario, Canada, 2016

(c)Abid Jan, 2016 


\section{AUTHOR'S DECLARATION FOR ELECTRONIC SUBMISSION OF A THESIS}

I hereby declare that I am the sole author of this thesis. This is a true copy of the thesis, including any required final revisions, as accepted by my examiners.

I authorize Ryerson University to lend this thesis to other institutions or individuals for the purpose of scholarly research.

I further authorize Ryerson University to reproduce this thesis by photocopying or by other means, in total or in part, at the request of other institutions or individuals for the purpose of scholarly research.

I understand that my thesis may be made electronically available to the public. 
Feasibility of CoMP Usage in Base Station Switching Off During Off-Peak Periods, Master of Applied Science, 2016, Abid Jan, Electrical and Computer Engineering, Ryerson University.

\begin{abstract}
Existing cellular networks remain operational throughout the year irrespective of traffic. The usage of Coordinated Multipoint (CoMP) transmission to provide service in the coverage area of a switched off base station (BS) during off-peak traffic hours has been investigated in this work.

The switching off of a BS reduces its energy consumption to zero, however to cover the switched off BS coverage area by neighbouring BS's, CoMP transmission causes an increase in energy consumption of the neighbouring BS's. With increasing the number of base stations taking part in CoMP transmission the power consumption of CoMP base stations and site air conditioning unit increases. Results show that the aggressive usage of CoMP is not feasible in most of the twelve switching modes investigated. From the Energy Efficiency Ratio the most energy efficient switching mode is identified. It is then applied to part of a cellular network and the amount of power saving and Carbon Dioxide equivalent $\left(\mathrm{CO}_{2} \mathrm{e}\right)$ is determined. It is found that within a network of 42 cells $7.26 \%$ power can be saved by switching off seven base stations during off-peak traffic hours.
\end{abstract}




\section{Acknowledgment}

I wish to acknowledge those who I feel have greatly aided me in completing this thesis. I would like to thank Professor Lian Zhao for providing an open study and discussion environment, having faith in me through out the project, constant encouragement and willingness to provide advice, and very clear guidance towards the success of this thesis. I am thankful to her for enlightening guidance, constructive suggestions, high-standard requirement and support. Special thanks go to my family especially my parents, brother, sister, wife and children Umar, Sara and Ali for their constant support. 


\section{Contents}

Author's Declaration . . . . . . . . . . . . . . . ii

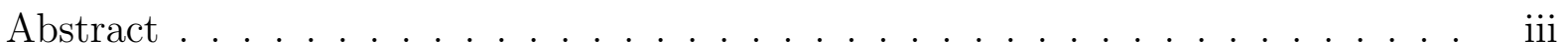

Acknowledgement ..................... iv

Table of Contents ......................

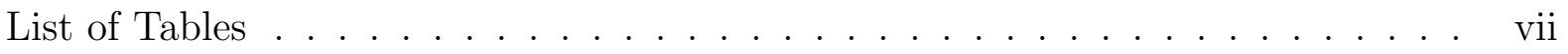

List of Figures . . . . . . . . . . . . . . . . . . viii

List of Appendices ..................... $\ldots \ldots$

1 Introduction 1

1.1 Background and Introduction $\ldots \ldots \ldots \ldots$

1.2 Literature Survey and Contributions . . . . . . . . . . . . . 3

1.3 Thesis Organization . . . . . . . . . . . . . . . . . . 7

2 Coordinated Multipoint Transmission in Base Station Switch Off Modes 9

2.1 Coordinated Multipoint Transmission . . . . . . . . . . . . . 9

2.2 Network Model . . . . . . . . . . . . . . . . . . . . . . . . . . . . . . . . 10

2.3 Cooperative Transmission in Switched Off Cell $C-0$ Coverage Area . . . . . . . . . . . . . . . . . 14

2.4 Selection of Switching Modes _ . . . . . . . . . . . . . . . . . 15

2.5 Worst Case Locations . . . . . . . . . . . . . . . . . . . . 16 
3 Power Consumption $\quad 36$

3.1 Introduction . . . . . . . . . . . . . . . . 36

3.2 Power Consumption Model . . . . . . . . . . . . . . . . . . . . . 37

3.3 Base Station Sectors Taking Part in CoMP Transmission in Each Switching

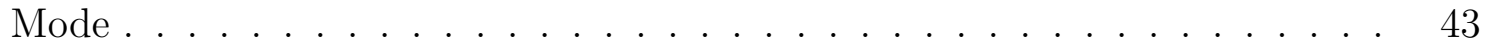

3.4 Analysis . . . . . . . . . . . . . . . . . . . . . 45

4 Numerical Results and Analysis $\quad 47$

4.1 SINR at Worst Case Locations . . . . . . . . . . . . . . . . . . . . . . 49

4.2 Effect of $N_{c}$ on Power Consumption of Base Stations Taking Part in CoMP

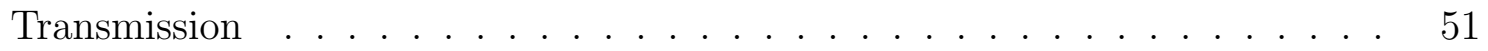

4.3 Comparision of BS Subsystems Power Requirement . . . . . . . . . . . 51

4.4 Energy Efficiency Ratio of Conventional and CoMP Feasible Switching Modes 53

4.5 Analysis of Mode No. 6 and Mode No. $7 \ldots \ldots 6$

4.6 Application in a Cellular Network . . . . . . . . . . . . . . 56

5 Conclusion $\quad 62$

5.1 Summary and Contributions . . . . . . . . . . . . . . . . . 62

5.2 Future Research Directions . . . . . . . . . . . . . . . . . . . 64

$\begin{array}{ll}\text { Appendices } & 65\end{array}$

$\begin{array}{lc}\text { A Abbreviation List } & 65\end{array}$

$\begin{array}{ll}\text { B Integration } & 66\end{array}$

$\begin{array}{ll}\text { Bibliography } & 69\end{array}$ 


\section{List of Tables}

2.1 Worst Case Location Distances in Mode No. $1 \ldots$. . . . . . . . . . . 18

2.2 Worst Case Location Distances in Mode No. 2 . . . . . . . . . . . . . . 19

2.3 Worst Case Location Distances in Mode No. 3 . . . . . . . . . . . . . 20

2.4 Worst Case Location Distances in Mode No. 4 . . . . . . . . . . . . . 23

2.5 Worst Case Location Distances in Mode No. 5 . . . . . . . . . . . . . . . . 23

2.6 Worst Case Location Distances in Mode No. 6 . . . . . . . . . . . . . . 26

2.7 Worst Case Location Distances in Mode No. 7 . . . . . . . . . . . . 26

2.8 Worst Case Location Distances in Mode No. $8 \ldots \ldots$. . . . . . . . . 27

2.9 Worst Case Location Distances in Mode No. 9 . . . . . . . . . . . . . 28

2.10 Worst Case Location Distances in Mode No. 10 . . . . . . . . . . . . 29

2.11 Worst Case Location Distances in Mode No. 11 . . . . . . . . . . . . 30

2.12 Worst Case Location Distances in Mode No. 12 . . . . . . . . . . . . . . 31

4.1 System Parameters for the Worst Case Locations . . . . . . . . . . . . . 48

4.2 System Parameters for the Power Consumption Model . . . . . . . . . . . . 48

4.3 User Capacity of Feasible Switching Modes . . . . . . . . . . . . . . . . 53

4.4 Base Station (Khar Bajur-NTR1P2138) Traffic During Night Times . . . . . 57

4.5 Base Station (Reshun-NTR1P4157) Traffic During Night Times . . . . . . 59 


\section{List of Figures}

1.1 Cellular Network Power Consumption $[5] \ldots \ldots$. . . . . . . . . . 2

1.2 Normalized Weekly Traffic Load of a Base Station $[13] \ldots \ldots$

2.1 Soft Frequency Reuse $[16] \ldots \ldots \ldots$

2.2 Mode No.1 . . . . . . . . . . . . . . . . . . . . . . . . . . . . 17

2.3 Mode No.2 . . . . . . . . . . . . . . . . . . . . . . . . . . . . . . . . . . . . . 19

2.4 Mode No.3 . . . . . . . . . . . . . . . . . . . . . . . . . . . . . . 21

2.5 Mode No.4. . . . . . . . . . . . . . . . . . . . . . . . . . . . . . . . . . . . . 22

2.6 Mode No.5 . . . . . . . . . . . . . . . . . . . . . . . . . . . . . . . . 24

2.7 Mode No.6 . . . . . . . . . . . . . . . . . . . . . . . . . . . . . . . . . . . . . 25

2.8 Mode No.7 . . . . . . . . . . . . . . . . . . . . . . . . . 27

2.9 Mode No.8 . . . . . . . . . . . . . . . . . . . . . . . . . . . . . . . . . . 28

2.10 Mode No.9 . . . . . . . . . . . . . . . . . . . . . . . . . . . . . 29

2.11 Mode No.10 . . . . . . . . . . . . . . . . . . . . . . . . . . 30

2.12 Mode No.11 . . . . . . . . . . . . . . . . . . . . . . . . . . . . . . 31

2.13 Mode No.12 . . . . . . . . . . . . . . . . . . . . . . . . . . . . . 32

3.1 Power Consumption of UMTS BS $[6] \ldots \ldots \ldots \ldots \ldots$

3.2 Power Consumption of a BS Site . . . . . . . . . . . . . . . . . . 39

4.1 SINR at Worst Case Locations . . . . . . . . . . . . . . . . . 50 
4.2 Change in Required Power with Number of BS Taking Part in CoMP Transmission . . . . . . . . . . . . . . . . . . . . . 52

4.3 Subsystems Power in Watts . . . . . . . . . . . . . . . . . 54

4.4 Power Consumption in Different Switching Modes to Provide Service in C-0 54

4.5 Bits per Joule of Feasible Modes . . . . . . . . . . . . . . . . . . . . 55

4.6 Extension of Mode No.7 in a Cellular Network . . . . . . . . . . . . . 58 


\section{List of Appendices}

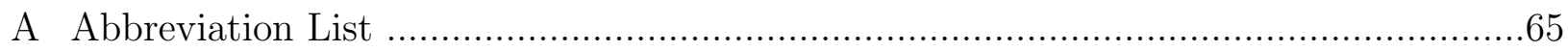

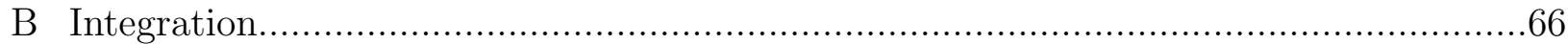




\section{Chapter 1}

\section{Introduction}

\subsection{Background and Introduction}

In recent years there has been tremendous growth in the Information Communication Technology (ICT) sector and the number of internet, data and cellular users have reached to billion of users. The number of cellular users have already surpassed the number of traditional landline phone users. The existing cellular networks which mainly consist of the Core Network (Mobile Service Switching Centres, Media Gateways, Signaling Gateways, Home Location Registers etc.) and the Access Network (Base stations, NodeB, eNodeB, Radio Network Controllers etc.) remain operationally on throughout the year. With the growth of number of cellular users and with bringing new geographical areas under coverage, the number of base stations (BS's) is also increasing, thereby resulting in an increase in energy consumption. For example the number of cellular subscribers which was 4.5 billion in 2012, is expected to each 7.6 billion by year 2020 [1]. According to [2] the number of connections (excluding machine to machine) shall reach 8 billion mark by year 2020 and the operators are constantly deploying new BS's to satisfy this growth. According to [2] the number of BS's have reached 4 million worldwide by year 2012 . 
With the increase in size of cellular networks electrical energy consumption is also increasing, although telecom equipment manufacturers are trying to reduce size of telecom equipments and thereby reduce energy consumption but the expansion in cellular networks is much greater than that effort resulting an overall increase in energy consumption.

There are different sources of electrical power generation i.e thermal, nuclear, hydro and other renewable sources for example solar and wind energy etc. In thermal power generation different types of fuel such as furnace oil, coal and natural gas is burnt to operate the thermal power plants (e.g steam and combined cycle power plants) for power generation. This burning of different types of fossil fuel is resulting in emission of Carbon Dioxide and Carbon Monoxide gases thereby polluting earth's environment and raising its temperature. The ICT sector contributed an estimated 830 million tons of Carbon Dioxide to the environment in year 2013 which was approximately $2 \%$ of the total global Carbon Dioxide emission [3].

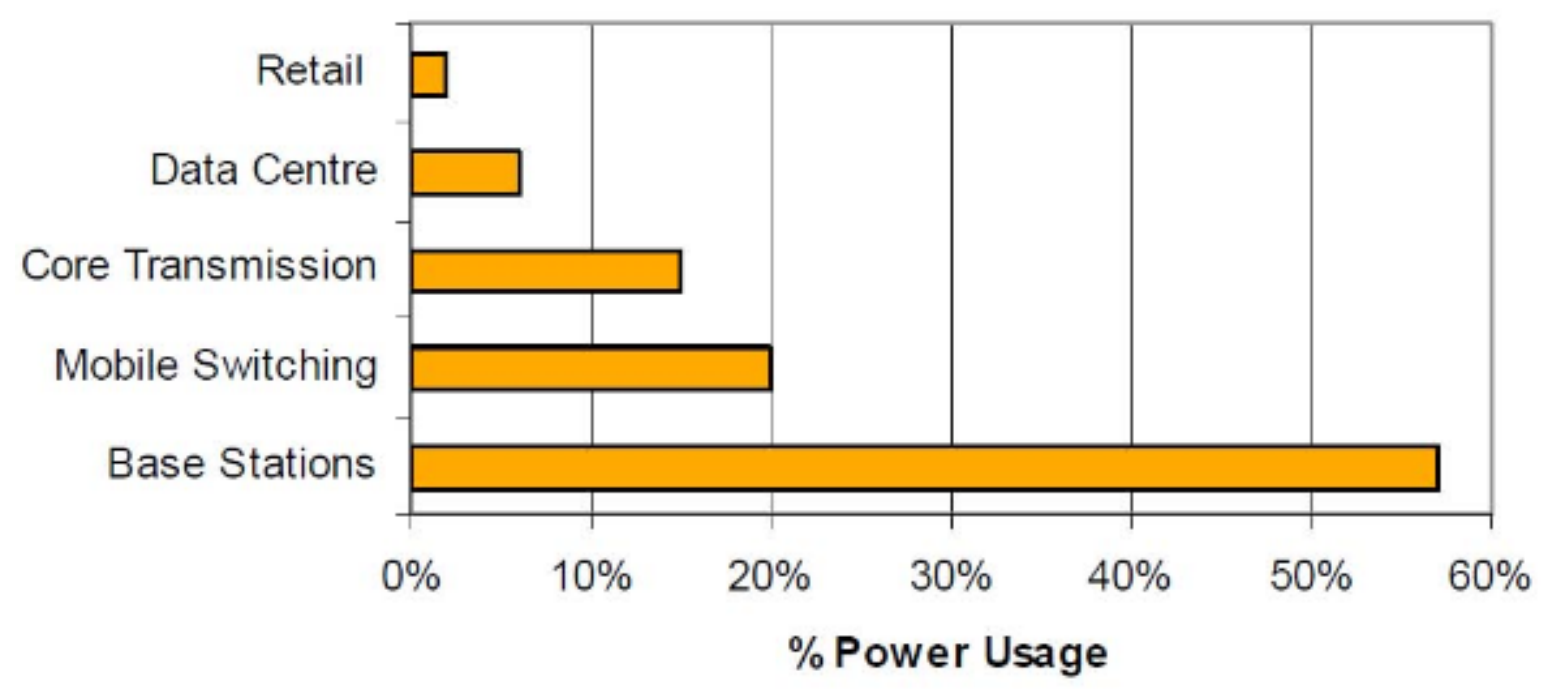

Figure 1.1: Cellular Network Power Consumption [5]

The increase in size of cellular networks also resulted in increase in energy consumption and in China alone the total energy consumption of the three main Chinese cellular operators 
reached 289 billion kilowatt-hours (kWh) in 2009 which was 25 percent more than in year $2008[4]$.

Within a cellular network the systems that are responsible for most of electrical energy consumption are base transceiver stations (BTS's) or simply BS. According to [5] 57\% of the energy consumption of a cellular network takes place in base station as shown in Fig. 1.1. Other equipments contributing to energy consumption at a BS site are backhauling system, alarm and monitoring system and air conditioning system.

\subsection{Literature Survey and Contributions}

Research efforts to reduce energy consumption in cellular access networks can be classified into three levels i.e Component Level, Link Level and Network Level [6]. Energy efficiency at Component Level can be achieved by increasing efficiency of High Power Amplifier (HPA), Low Noise Amplifier (LNA), DC/DC convertor and rectifier. Efficiency of amplifiers can be increased by using Doherty amplifiers or using special materials for power amplifier transistors. Reduction in power consumption of signal processing units in base stations can be achieved using ASIC and FPGA architectures [11]. Another way of reducing energy consumption at Component Level is by placing Radio Frequency Unit (RFU) near the antenna thereby reducing the losses in feeder cable[12]. This type of RFU is known as Radio Resource Unit (RRU).

At the Link Level discontinous transmission in which some of the hardware components are switched off instead of turning off the whole base station, increases energy efficiency. Different sleep modes such as micro sleep mode in which base stations suspend transmission in the order of milliseconds and deep sleep modes in which transmission is shut down for extended period of time, have been investigated by researchers.

According to [13] and as shown in Fig. 1.2 the base station traffic within a cellular network 
changes dynamically with much less traffic during night times, on Saturdays, Sundays and other holidays. Therefore the base stations are under utilized during these times.

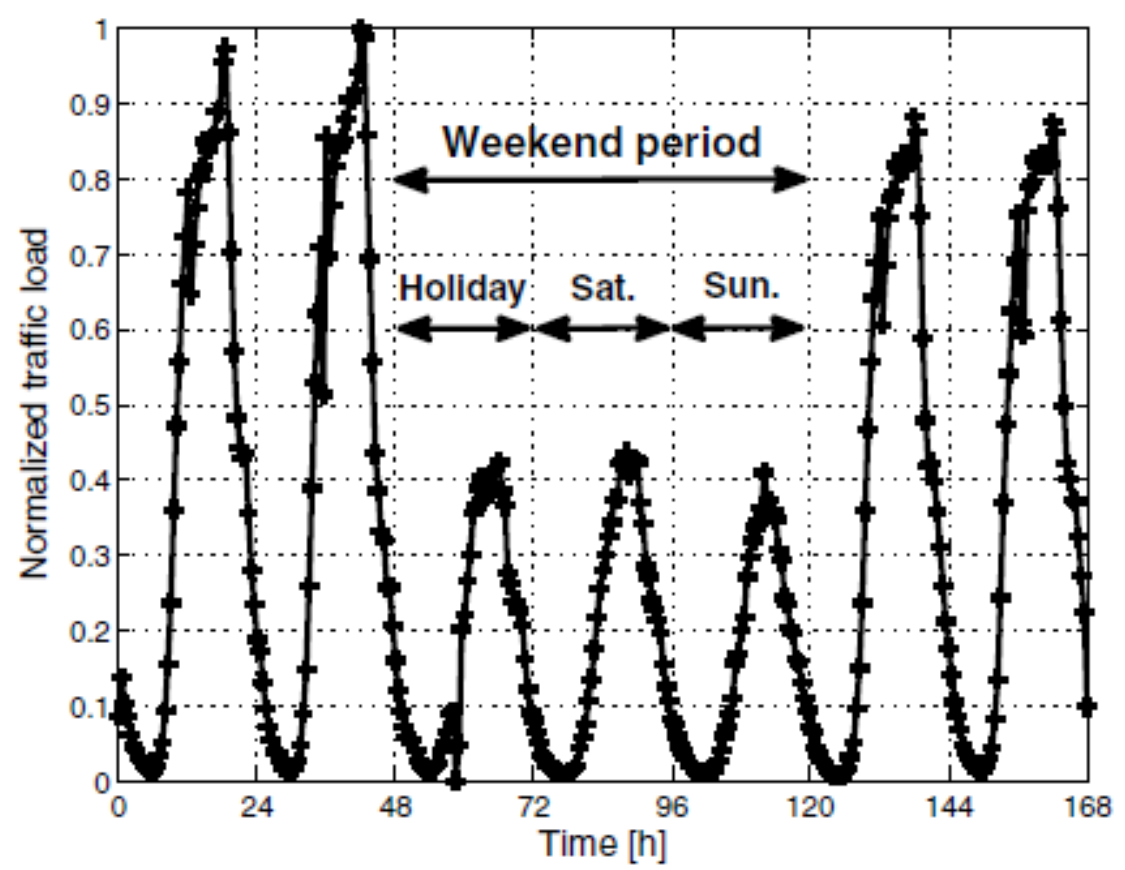

Figure 1.2: Normalized Weekly Traffic Load of a Base Station [13]

Based on this phenomenon of 24 hours traffic pattern an approach has been advocated in recent literature, to turn off some base stations in a cellular network which are experiencing low traffic (in night and in holiday times) in order to reduce energy consumption in the network.

According to that approach in order to reduce energy consumption, one or more base stations having traffic below a certain threshold for a certain time period, can be turned off. The selection of a certain time period of base station to remain under utilized shall prevent its frequent On/Off operation which is not desirable. The neighbouring base stations can be used to provide service in the switched off cell area, provided the neighbouring base stations have enough spare capacity and do not experience any congestion at Network Level. 
However many considerations are being ignored while advocating for this approach of turning off some of the base stations. For example the increase in interference in the network due to increase in transmit power by neighbouring BS's to cover the switched off area, ensuring the QoS in the switched off area as it was before since the BS- $U E$ distance is now more, the availability of spare capacity in the neighbouring BS's and the increase in power consumption due to the increased processing in $\mathrm{BS}$ as a result of using cooperation among BS's. The increase in BS energy consumption due to increase in its transmit power has also been ignored in previous work.

In [7] cooperation between different competing operators in the same geographical area has been discussed for saving energy and accordingly this cooperation can reduce energy consumption by 20\%. In [7] it has been suggested that at low traffic times base station of one operator can be turned off while the other operator shall keep its base station operationally on in that particular coverage area and provide service to its own as well as to the users of the other network operator whose base station has been turned off. However this approach shall be feasible only if the coverage areas of both network operators are completely overlapping. But in reality complete overlapping among different operators throughout the licensed area seldom occurs. Besides providing an exact QoS to the roaming users from the switched off cells of one operator to the network operator whose base stations are operationally on, may not be ensured.

In [10] the energy consumption reduction using a dynamic management of network resources i.e turning off a base station during low traffic and providing service in the area of switched off base stations, by the neighbouring base stations has been investigated.

The application of cell zooming technique in which the base station varies its coverage area according to traffic load is investigated in [8]. Implementation of cell zooming in mobile networks shall need new components and corresponding functionalities. In order to implement cell zooming the suggested technique in [8] is to adjust the physical parameters such 
as increasing and decreasing the transmit power of base station. Other suggestions include physical adjustment of antenna height and antenna tilt of base station which shall need additional mechnical instrument to carry out such adjustment.

When a base station is turned off then in order to avoid coverage holes in the switched off base station area, it is not clear how much increase in transmit power is needed by the nearby neighbouring base stations. The increase in transmit power by the neighbouring base stations shall cause an increase in interference and thereby reduce capacity of the effected cells. Contrary to that according to [23] one third of operating energy could be saved by reducing the transmission power of base stations. Therefore any increase in transmit power is going to increase energy consumption of the operational base station.

CoMP (Coordinated Multipoint) transmission is very useful to increase the spectrum efficiency and data rates in wireless communication and its usage in green communication has been discussed in [9], but no detailed analysis on its energy efficiency such as bits per Joule has been done. For example in Cooperative Beamforming (CBF) the base station forms radio beams to enhance the signal strength to serve the cell edge users while forming null steering towards users in its neighbouring cells resulting less transmission power to serve the intended users. However the increase in energy consumption due to CBF for example additional energy consumption in channel estimation, sharing of channel information and sharing of source message, have not been given considerations.

In [18] in order to cover the coverage area of the switched off BS's and to compensate the loss due to increased distance between the user equipment $(U E)$ and the BS, CoMP transmission by the neighbouring BS's surrounding the switched off cell, is considered. However the effect of interference on extending the signal to the users at worst case locations has not been taken into account.

In this work the feasibility of base stations switching off for reducing energy consumption of the cellular network is investigated. The effect of interference and frequency planning has 
been taken into account. Besides the increase in energy consumption due to baseband signal processing, due to backhauling as a result of using CoMP transmission and a subsequent increase in air conditioning energy consumption and in DC/DC converter have been taken into considerations.

A power consumption model for BS's taking part in CoMP transmission to provide service in a particular switched off BS area is developed. The earlier models were applicable to $2^{\text {nd }}$ and $3^{\text {rd }}$ generations of cellular networks only and this model can be applied to $4^{\text {th }}$ generation and latter cellular networks.

The effect of changing the number of base stations taking part in CoMP transmission on the power consumption of the BS site is investigated. The worst case locations within the switched off area for different combinations of BS's using CoMP transmission are used as a reference for deciding the feasibilty of a particular switching mode and required QoS. In earlier works only the energy saving has been discussed without giving any considerations to QoS within the switched off area.

The research work is further extended and is applied to a network. The saving in power and operational cost can be calculated for the whole cellular network.

\subsection{Thesis Organization}

The remaining of the thesis is organized as follows:

- Chapter 2: In this chapter the use of CoMP to provide service in the switched off cell coverage area is investigated. The worst case locations of each of the twelve switching modes are used as a reference point. If the QoS is ensured at the worst case locations then we can say that QoS is ensured at all other locations which are much closer to the base stations taking part in CoMP transmission in the switched off area. In this chapter the feasible switching modes are identified. As compared to earlier work the desired 
QoS before and after BS switching off is ensured. The user within the switched off cell is free to roam and the useful signal strength varies within the same cell, therefore the expected value of SNR is found and the corresponding per user capacity is calculated for each of the feasible switching modes.

- Chapter 3: In this chapter a power consumption model of a base station site is developed. This model shows the power consumption of a BS taking part in CoMP transmission. CoMP has been included in LTE-Advanced Release 11 and the power consumption model presented can be used to find out the power consumed in various subsystems of a BS providing service in the coverage area of a neighbouring switched off BS. The power consumption model can be used to investigate the effect of number of BS's taking part in CoMP on the power required for various systems in a BS site (e.g air conditioning, bakhauling, rectifier etc). As compared to earlier power consumption models which were applicable to the stand alone operation of $2 \mathrm{G}$ and $3 \mathrm{G}$ BS, the model developed in this chapter is applicable to $4 \mathrm{G}$ and latter networks using CoMP transmission. As compared to earlier work the increase in energy consumption of the neighbouring BS's to cover the switched off cell area has been taken into account.

- Chapter 4: In this chapter numerical results are presented. The feasibility, power required to cover a switched off BS area and Energy Efficiency Ratio (EER) of different feasible switching modes is discussed. The effect of the number of cooperating BS on the power consumption is discussed. In this chapter the power requirement for internal subsystems of a BS operating in CoMP mode is also discussed. The research work is then applied to a cellular network and the power saved is calculated. Based on these results the Carbon Dioxide equivalent $\left(\mathrm{CO}_{2} e\right)$ of the energy saved is calculated.

- Chapter 5: This chapter concludes this thesis and future research directions are discussed. 


\section{Chapter 2}

\section{Coordinated Multipoint Transmission in Base Station Switch Off Modes}

\subsection{Coordinated Multipoint Transmission}

Coordinated Multipoint Transmission (CoMP) has been included in LTE-Advanced Release 11 [14]. CoMP inclusion in LTE-Advanced is to improve cell edge users data rates. In different research papers such as [18] and [28] CoMP has been used to provide service in the area of a base station that is switched off due to low traffic load during holidays, night or other low traffic hours of a day. Since the user distances in the switched off cell area increase from all the neighbouring base stations, therfore in order to provide service to those users located in the swtiched off cell area, the neighbouring base station or a number of neighbouring base stations to which these users need to be handed over, have either to increase transmit power or use CoMP to compensate for the increased distance of the users in the switched off cell area from the serving base station(s). In [19] co-channel interference has not been taken into account, besides the increase in power consumption due to increased signal processing in base station due to Cooperative Multipoint Transmission has not been 
taken into account. In this work both these factors have been taken into account.

The other method to provide service in the coverage area of a switched off cell is to increase the transmit power of the neighbouring base stations. However increasing the transmit power of a base station during low traffic hours increases interference in other operationally on neighbouring base stations thereby effecting the QoS in those base stations coverage areas.

In [15] the authors have investigated whether sleeping cell sites experiencing low traffic and allowing neighboring cell sites to cover the geographical area served by the sleeping cell can be viable or not. It has been shown in [15] that sleeping cell sites may not necessarily be viable or energy efficient.

Another approach in the literature is to use CoMP by neighbouring base stations to provide coverage in the switched off base station area.

In the following sections the use of CoMP shall be investigated to find out whether the neighbouring base stations shall be able to ensure the QoS at the worst case locations of a user in the switched off cell coverage area as it was at the same location when the base station was not turned off. If QoS is satisfied at worst case locations then it shall be satisfied at all other locations much closer to the base stations taking part in CoMP transmission.

In this work different number of neighbouring base stations (depending on whether they shall be able to take part in cooperation or not which further depends upon their traffic load and spare capacity) shall be used. Neighbouring base stations having traffic load less than a specific threshold can take part in Coordinated Multipoint transmission. This traffic threshold shall be decided by the concerned cellular operator.

\subsection{Network Model}

Consider a cellular network consisting of $N$ base stations and the coverage area of each base station is represented by a hexagonal cell, each of radius $R$. The set of base stations 
$B=\left\{B_{0}, B_{1}, B_{2} \ldots, B_{N}\right\}$ lies within a two diamensional area $A$.

In this network soft frequency is used (a frequency reuse factor of 1) in the central region of all the cells and a frequency reuse factor of 3 is used at the outward cell region near the boundaries of each cell. This results into reduction of interference. The use of soft frequency reuse scheme is shown in Fig. 2.1 [16].

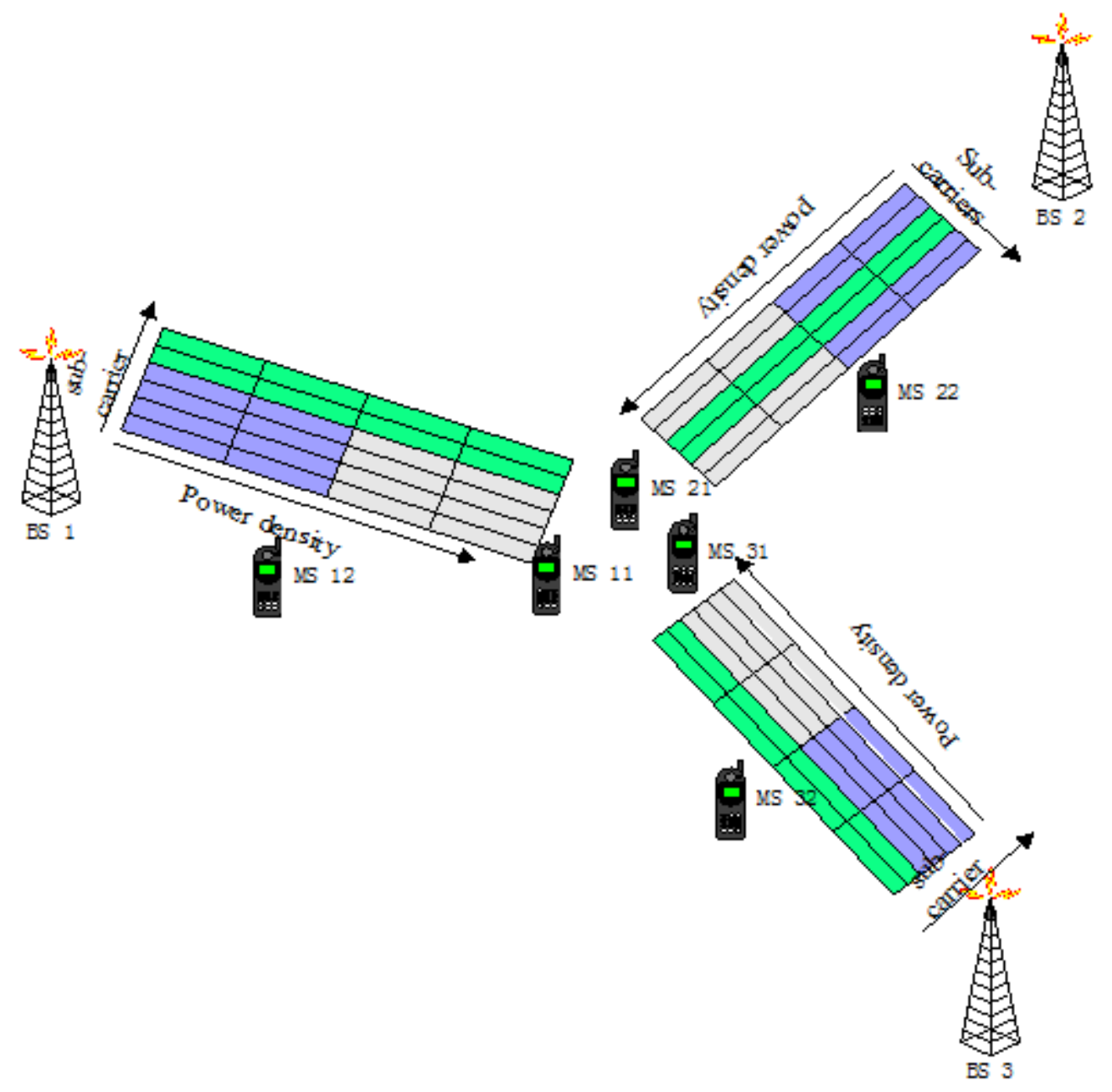

Figure 2.1: Soft Frequency Reuse [16]

The coverage area of the base station in the centre of the network is represented by cell $C-0$ and its immediate six neighbouring base stations coverage areas are represented by cells $C-1, C-2, C-3, C-4, C-5$ and $C-6$. The coverage areas of the next tier of 
neighbouring base stations are represented by cells $C-1^{\prime}, C-2^{\prime}, C-3^{\prime}, C-4^{\prime}, C-5^{\prime}$, $C-6^{\prime}, C-7^{\prime}, C-8^{\prime}, C-9^{\prime}, C-10^{\prime}, C-11^{\prime}, C-12^{\prime}$ respectively as shown in Fig. 2.2. The second tier of cells are included to take interference effect into considerations in the switched off cell coverage area. Each base station located at the centre of the corresponding cell is equiped with two antennas to make use of MIMO technology.

Two interference mitigation techniques available in the literature are

- Active Approach

- Passive Approach

Active approach is further categorized into

- Interference Avoidance

- Use of Smart Antenna

Interference avoidance can be categorized into

- Flexible Frequency Reuse (FFR)

- Dynamic Channel Allocation (DCA)

The usage of smart antenna can be categorized into Beamforming and Distributed Antenna. The Passive Approach of interference mitigation technique can be categorized into interference cancellation and interference suppression (e.g maximum ratio combining and minimum mean square error).

It is assumed that the base stations have the same maximum transmit power $P_{t x}$ and each base station is connected with its neighbouring base station set $N_{b}$ via a dedicated backhaul link through microwave links. 
A hexagonal cell structure has been considered to represent the cellular network with the assumption that the base station is located at the centre of the hexagon. We focus on downlink communication. The base stations are equipped with three sectorized antennas and each sector is using MIMO technology with $M=2$.

The mobile users represented by $U E s$ are uniformly distributed within the network during peak traffic hours and the traffic is following the typical day night phenomenon as shown in Fig. 1.2. The base stations are located at equal distances and each base station has a certain capacity represented by $C$ beyond which no new call request can be admitted to avoid congestion and call failures.

The base station having a normalized traffic load of less than or equal to a certain traffic threshold can be switched off. The switched off base station coverage area can be served by the neighbouring base stations having enough spare capacity and using coordinated transmission in the switched off base station coverage area. The minimum traffic is when there is only one user in the coverage area of the cell. The maximum number of base stations that can take part in Coordinated Multipoint transmission is six in this work and the minimum number is equal to one.

The effectiveness of this cooperation shall be evaluated in the subsequent sections by finding the signal to interference plus noise ratio (SINR) at locations in the switched off base station coverage areas that are located farthest away from the cooperative base stations, such locations are referred to as worst case locations in this work and are represented by ' $X$ ' for switching Modes 1 to 12 shown in Fig. 2.2 to Fig. 2.13. 


\subsection{Cooperative Transmission in Switched Off Cell $C-0$ Coverage Area}

During 24 hours period of a day some base stations of a cellular network can be turned off in order to save energy. The users traffic in switched off base station coverage area can be handed over to the neighbouring base stations leading a higher aggregated traffic load for those base stations. Due to increase in distance of users in coverage area of switched off base station from the neighbouring base stations, the signal strength and subsequently the SINR of the user shall be reduced as compared to the SINR when the base station was not turned off. In order to overcome the issue of reduced SINR the concept of Coordinated Multipoint transmission is used to compensate for the decrease in signal strength when a base station is turned off. According to [17], [18] two Coordinated Multipoint transmission schemes have been included in LTE-Advanced i.e

- Coordinated Scheduling

- CoMP Joint Processing

In Coordinated Scheduling, mobile user data is available in one base station which is called the Master base station but user scheduling and beam forming decisions are made with coordination among neighbouring base stations. In CoMP Joint Processing user data to be transmitted to one mobile user is available in multiple neighbourng base stations of the network. CoMP joint transmission is a subclass of joint processing where data channel to one user terminal is simultaneously transmitted from multiple base stations. In [19], Coordinated Multipoint transmission has been used to provide service in the coverage area of a switched off cell but interference by the neighbouring cells have not been taken into account. In this work, interference by the base stations in the first tier and second tier have been taken into account while providing service in the switched off cell coverage area. The twelve switching 
off modes identified in that reference shall be used while taking into account the co-channel interference. Besides in [19] the additional baseband signal processing power consumption due to CoMP among the base stations is considered to be negligible, however in this work the increased power consumption due to CoMP has been taken into account in the next chapter. According to [20] the SINR in a multi-cell MISO cooperative cellular network with co-channel interference is given by

$$
S I N R=\frac{\sum_{c b=1}^{N_{b}} \sum_{j=1}^{N_{t}^{c}} \frac{1}{r_{c b}^{4}}\left|z_{c b, j}\right|^{2}}{N_{0}+\sum_{b=1}^{\infty} \sum_{j=1}^{N_{t}} \frac{1}{r_{b}^{4}}\left|z_{b, j}\right|^{2}},
$$

whereas $N_{0}$ is the noise power spectral density, $c b$ represents the number of cooperative base stations, $j$ is the number of antennas at base station site, $r_{c b}$ is the distance of the cooperative base station from the mobile user, $r_{b}$ is the distance of the interfering base station from the mobile user, $z_{c b, j}$ and $z_{b, j}$ are random variables following the Nakagami$\mathrm{m}$ distribution and represents signal passing through the Nakagami-m fading channel from antenna $j$ of the cooperative base station $c b$ and interfering base station $b$ respectively. Each signal passing through the Nakagami fading channel is assumed to follow an i.i.d complex Gaussian distribution with zero mean and variance of 1 . Path loss exponent $\alpha$ is taken as 4 .

\subsection{Selection of Switching Modes}

Based on base station day night traffic phenomenon the central BS having coverage area $C-0$ is selected to be turned off. There are six neighbouring base stations in first tier as shown in Fig. 2.2. The selection of the number of base stations taking part in CoMP transmission in the coverage area of the switched off cell $C-0$ is based on the availability of spare capacity in base stations of first tier. In Mode No. 1 shown in Fig. 2.2 six base 
stations in first tier have enough spare capacity to provide service to users in switched off cell $C-0$. In Mode No. 2 as shown in Fig. 2.3 base station identified by cell $C-4$ is not taking part in CoMP transmission in the switched off area $C-0$ due to the unavailability of spare capacity to provide service to users in the switched off area. Similarly in Mode No.12 there is only one base station identified by cell $C-1$ in first tier providing service in the switched off cell $C-0$ coverage area and the rest of the five base stations in first tier i.e $C-2, C-3, C-4, C-5$ and $C-6$ are not taking part in CoMP transmission due to the unavailability of spare capacity in those base stations.

\subsection{Worst Case Locations}

For comparing the QoS of base station $B_{0}$ when it is providing service in its own coverage area $C-0$ i.e conventional operation, to the situation when BS $B_{0}$ is turned off and the neighbouring BS's of $B_{0}$ use CoMP to provide service in the switched off area $C-0$, it is necessary to find out the worst case locations. The number and locations of the neighbouring cooperative base stations in each switching mode have been taken into consideration in determining the worst case locations. Fitting the geographical locations of base stations and user in a two dimensional coordinate system, both have been assigned a pair of coordinates. The worst case location within the hexagonal cell of the central cell $C-0$ is equivalent to solving the following constrained minimization problem [19],

$$
\begin{aligned}
& \min _{x, y} \quad f(x, y)=\sum_{k=1}^{K} \frac{1}{\left[\left(x-x_{k}\right)^{2}+\left(y-y_{k}\right)^{2}\right]^{\frac{\alpha}{2}}} \\
& \text { s.t. }\left\{\begin{array}{c}
-\sqrt{3} R \leq \sqrt{3} x+y \leq \sqrt{3} R ; \\
-\sqrt{3} R \leq \sqrt{3} x-y \leq \sqrt{3} R ; \\
-\frac{\sqrt{3}}{2} R \leq y \leq \frac{\sqrt{3}}{2} R,
\end{array}\right.
\end{aligned}
$$


whereas $(x, y)$ and $\left(x_{k}, y_{k}\right)$ are the location coordinates of $U E$ and the $k^{\text {th }}$ BS respectively. The worst case points have been found from the Karush-Kuhn-Tucker conditions. The objective function shall be minimum if the denominator of above equation is maximum. These locations are represented by ' $X$ ' identified in cell $C-0$ i.e the cell representing the BS to be turned off due to its extremely low traffic. The worst case locations of different switching modes are located such that the received signal value shall be the lowest as compared to all other locations within the coverage area of the switched off cell for each mode under consideration. Using Law of Cosines and Pythagorean theorem the distance of the worst

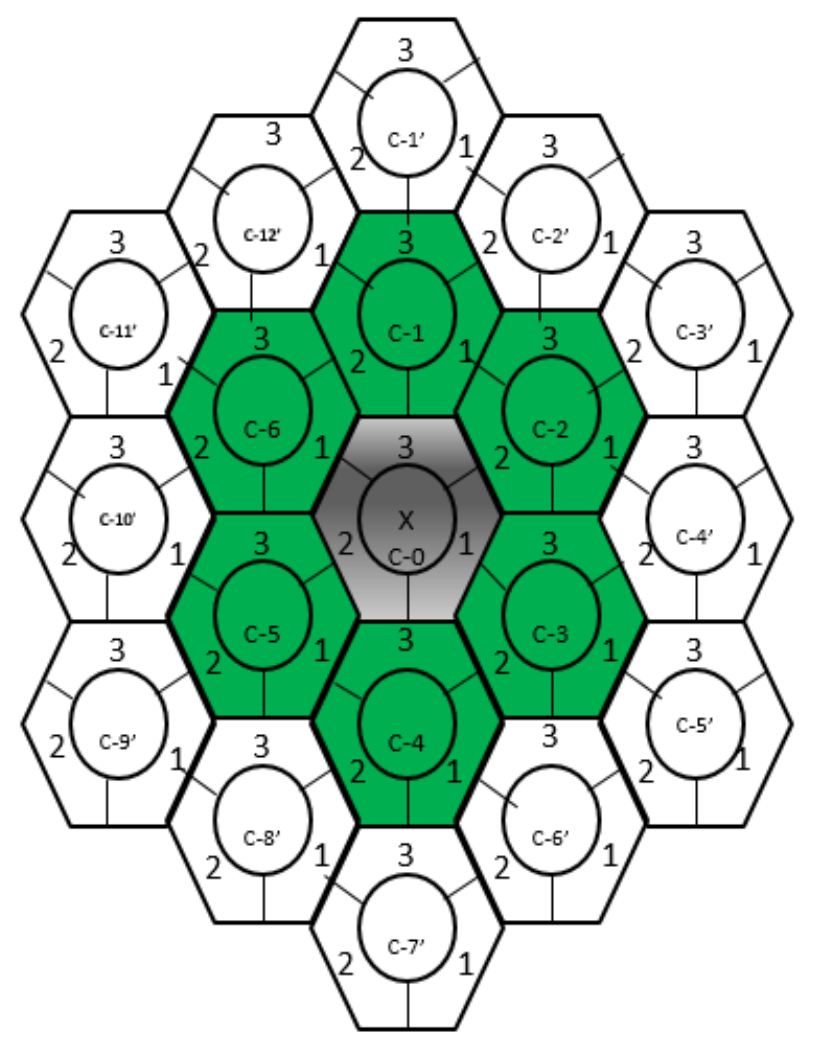

Figure 2.2: Mode No.1

case locations in each of the twelve switching modes are calculated for the base stations taking part in cooperative transmission and for the base stations which are acting as a source of interference for mobile user at those worst case locations. Corresponding distances 
of these worst case locations from the base stations taking part in cooperative transmission and from the base stations which are acting as a source of interference are shown in Table 2.1 - Table 2.12 .

Table 2.1: Worst Case Location Distances in Mode No. 1

\begin{tabular}{|l|l|l|l|}
\hline \hline $\begin{array}{l}\text { BS } \\
\text { (CoMP })\end{array}$ & $\begin{array}{l}\text { Worst Case Location } \\
\text { Distance }\end{array}$ & Interferers & $\begin{array}{l}\text { Worst Case Location } \\
\text { Distance }\end{array}$ \\
\hline$C-1$ & $1.73 R$ & $C-1^{\prime}$ & $3.46 R$ \\
$C-2$ & $1.73 R$ & $C-2^{\prime}$ & $3.00 R$ \\
$C-3$ & $1.73 R$ & $C-3^{\prime}$ & $3.46 R$ \\
$C-4$ & $1.73 R$ & $C-4^{\prime}$ & $3.00 R$ \\
$C-5$ & $1.73 R$ & $C-5^{\prime}$ & $3.46 R$ \\
$C-6$ & $1.73 R$ & $C-6^{\prime}$ & $3.00 R$ \\
& & $C-7^{\prime}$ & $3.46 R$ \\
& & $C-8^{\prime}$ & $3.00 R$ \\
& & $C-9^{\prime}$ & $3.46 R$ \\
& & $C-10^{\prime}$ & $3.00 R$ \\
& & $C-11^{\prime}$ & $3.46 R$ \\
& & $C-12^{\prime}$ & $3.00 R$ \\
\hline
\end{tabular}

Considering $R$ to be the radius of the hexagon. Then the distance from the centre of any of the cells i.e $C-1$ to $C-6$ to the centre of the central cell $C-0$ is

$$
\frac{\sqrt{3} R}{2}+\frac{\sqrt{3} R}{2} \approx 1.73 R
$$

The distance from the centre of any of the hexagonal cell to the mid point on any of its six sides is $\frac{\sqrt{3}}{2} R$. The distance of the worst case location from the centre of the interfering cells $C-1^{\prime}, C-3^{\prime}, C-5^{\prime}, C-7^{\prime}, C-9^{\prime}$ and $C-11^{\prime}$ is $4\left(\frac{\sqrt{3}}{2}\right) R$. The corresponding distances from the interfering cells $C-2^{\prime}, C-4^{\prime}, C-6^{\prime}, C-8^{\prime}, C-10^{\prime}$ and $C-12^{\prime}$ is $3 R$. These distances are shown in Table 2.1. 


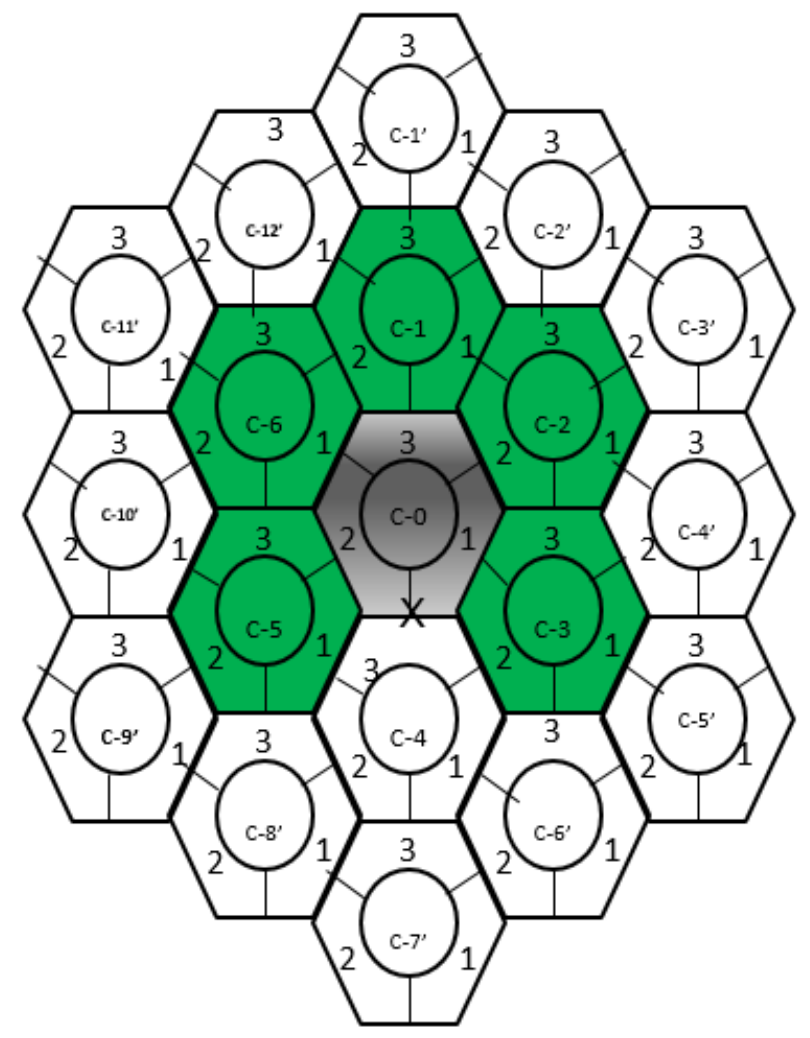

Figure 2.3: Mode No.2

Table 2.2: Worst Case Location Distances in Mode No. 2

\begin{tabular}{|l|l|l|l|}
\hline \hline BS & Worst Case Location & Interferers & $\begin{array}{l}\text { Worst Case Location } \\
\text { Distance }\end{array}$ \\
\hline$C-1$ & Distance & & $4.33 R$ \\
$C-2$ & $2.60 R$ & $C-1^{\prime}$ & - \\
$C-3$ & $1.50 R$ & $C-9^{\prime}$ & $3.12 R$ \\
$C-5$ & $1.50 R$ & $C-10^{\prime}$ & $3.12 R$ \\
$C-6$ & $2.29 R$ & $C-11^{\prime}$ & $3.96 R$ \\
\hline
\end{tabular}

In Mode No.2 the distance of the cell $C-1$ from the worst case location is

$$
3\left(\frac{\sqrt{3}}{2}\right) R \approx 2.60 R
$$


Cell $C-2$ distance from the worst case location is found as

$$
\sqrt{(1.5 R)^{2}+\left(2 \cdot \frac{\sqrt{3}}{2} R\right)^{2}} \approx 2.29 R .
$$

Distance of interfering cell $C-1^{\prime}$ to the worst case location of Mode No.2 is

$$
5\left(\frac{\sqrt{3}}{2}\right) R \approx 4.33 R .
$$

In case of cell $C-9^{\prime}$ the worst case distance is

$$
\sqrt{(3 R)^{2}+\left(\frac{\sqrt{3}}{2} R\right)^{2}} \approx 3.122 R .
$$

Distance of cell $C-11^{\prime}$ is calculated as

$$
\sqrt{(3 R)^{2}+\left(3 \cdot \frac{\sqrt{3}}{2} R\right)^{2}} \approx 3.96 R .
$$

Table 2.3: Worst Case Location Distances in Mode No. 3

\begin{tabular}{|l|l|l|l|}
\hline \hline BS & Worst Case Location & Interferers & $\begin{array}{l}\text { Worst Case Location } \\
\text { Distance }\end{array}$ \\
\hline$C-1$ & Distance & & $3.12 R$ \\
$C-3$ & $1.50 R$ & $C-1^{\prime}$ & 3.32 \\
$C-4$ & $2.29 R$ & $C-9^{\prime}$ & $4.33 R$ \\
$C-6$ & $2.29 R$ & $C-10^{\prime}$ & $3.77 R$ \\
& & $C-11^{\prime}$ & $3.96 R$ \\
\hline
\end{tabular}

In case of Mode No.3 two worst case locations are possible as shown in Fig. 2.4. For the worst case location on the right, cell $C-1$ and $C-3$ are at equal distance of 1.5R. Each of the interior angles of a hexagon is 120 degree. Using Law of Cosines, cells $C-4$ and $C-6$ 


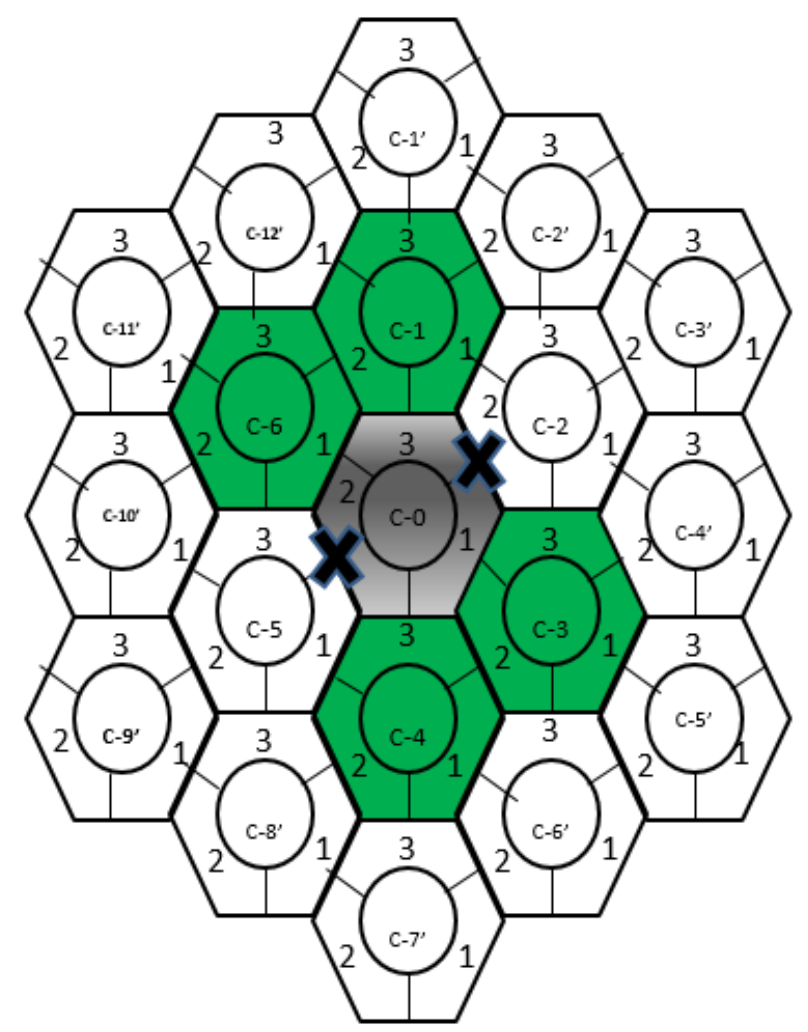

Figure 2.4: Mode No.3

distance from the worst case location on the right in Fig. 2.4. can be determined as follows

$$
\sqrt{(2 R)^{2}+(0.5 R)^{2}-2(2 R)(0.5 R) \cos \left(\frac{2 \pi}{3}\right)} \approx 2.29 R .
$$

In Mode No.4 as shown in Fig. 2.5. the cells taking part in CoMP transmission in the area of the switched off cell $C-0$ are $C-1, C-2, C-3$ and $C-4$. Cells $C-1$ and $C-4$ each have a distance of $2 R$ from the worst case location. Cells $C-2$ and $C-3$ each have distance from the worst case location equal to

$$
\sqrt{(2.5 R)^{2}+\left(\frac{\sqrt{3}}{2} R\right)^{2}} \approx 2.64 R .
$$




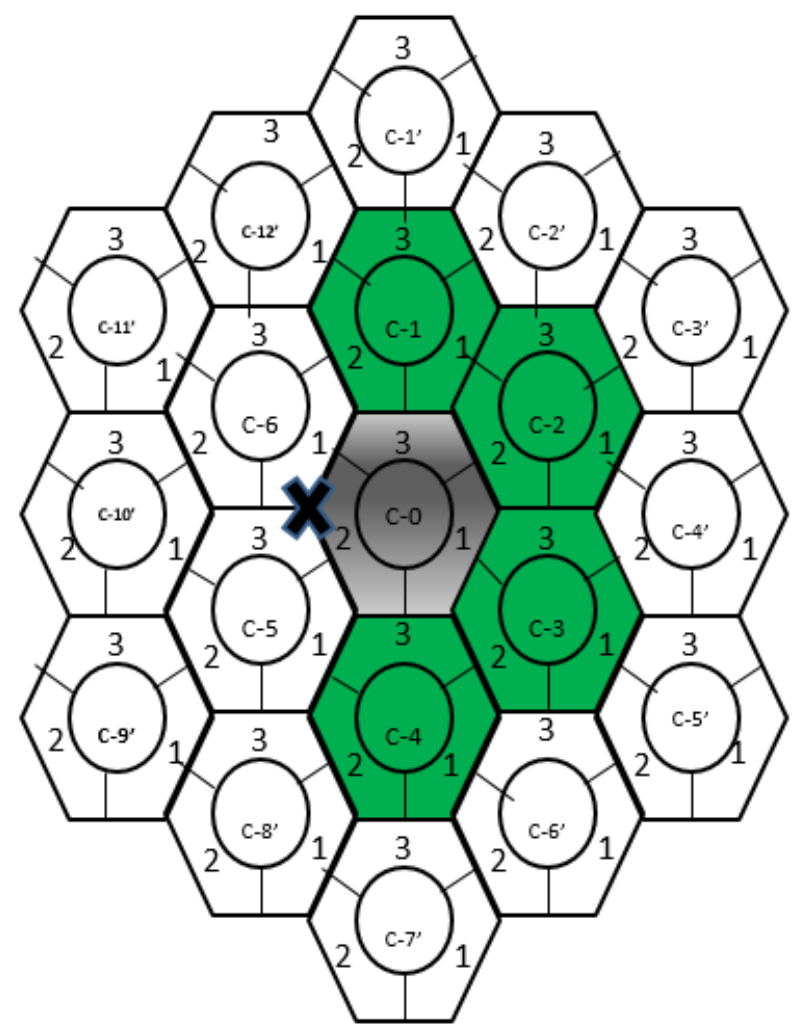

Figure 2.5: Mode No.4

The interfering cells in Mode No. 4 are $C-1^{\prime}, C-2^{\prime}, C-3^{\prime}, C-4^{\prime}$ and $C-5^{\prime}$. For interfering cell $C-1^{\prime}$ the worst case location distance is caclulated as

$$
\sqrt{(R)^{2}+\left(4 \cdot \frac{\sqrt{3}}{2} R\right)^{2}} \approx 3.60 R .
$$

Similarly for other interfering cells the worst case location distances are calculated and tabulated in Table 2.4 .

In case of Mode No.5 shown in Fig. 2.6 there are four cells taking part in CoMP transmission i.e $C-1, C-2, C-4$ and $C-6$. The interfering cells are $C-3, C-5$ and cells $C-1^{\prime}$ to $C-12^{\prime}$.

In Mode No.6 shown in Fig. 2.7 the cells taking part in useful transmission to the user 
Table 2.4: Worst Case Location Distances in Mode No. 4

\begin{tabular}{|c|c|c|c|}
\hline $\begin{array}{l}\mathrm{BS} \\
(\mathrm{CoMP})\end{array}$ & $\begin{array}{l}\text { Worst Case Location } \\
\text { Distance }\end{array}$ & Interferers & $\begin{array}{l}\text { Worst Case Location } \\
\text { Distance }\end{array}$ \\
\hline$C-1$ & $2.00 R$ & $C-1^{\prime}$ & $3.60 R$ \\
\hline$C-2$ & $2.64 R$ & $C-2^{\prime}$ & $3.60 R$ \\
\hline$C-3$ & $2.64 R$ & $C-3^{\prime}$ & $4.36 R$ \\
\hline$C-4$ & $2.00 R$ & $C-4^{\prime}$ & $4.00 R$ \\
\hline & & $C-5^{\prime}$ & $4.36 R$ \\
\hline
\end{tabular}

Table 2.5: Worst Case Location Distances in Mode No. 5

\begin{tabular}{|l|l|l|l|}
\hline \hline BS & Worst Case Location & Interferers & $\begin{array}{l}\text { Worst Case Location } \\
\text { Distance }\end{array}$ \\
\hline$C-1$ & Distance & & $1.64 R$ \\
$C-2$ & $1.92 R$ & $C-3$ & $1.64 R$ \\
$C-4$ & $1.83 R$ & $C-5$ & $3.65 R$ \\
$C-6$ & $1.54 R$ & $C-1^{\prime}$ & $3.16 R$ \\
& $1.83 R$ & $C-2^{\prime}$ & $3.56 R$ \\
& & $C-3^{\prime}$ & $3.00 R$ \\
& & $C-4^{\prime}$ & $3.37 R$ \\
& & $C-5^{\prime}$ & $2.83 R$ \\
& & $C-6^{\prime}$ & $3.28 R$ \\
& & $C-7^{\prime}$ & $2.83 R$ \\
& & $C-8^{\prime}$ & $3.37 R$ \\
& & $C-9^{\prime}$ & $3.00 R$ \\
& & $C-10^{\prime}$ & $C-11^{\prime}$ \\
& & $C-12^{\prime}$ & $3.56 R$ \\
\hline
\end{tabular}

located at the worst case location are $C-1, C-3$ and $C-5$ each having distance of

$$
2\left(\frac{\sqrt{3}}{2}\right) R \approx 1.73 R
$$

The interfering base stations are represented by cells $C-2, C-4, C-6$ and cells $C-1^{\prime}$ to $C-12^{\prime}$. The interfering cells $C-2, C-4$ and $C-6$ each have distance of $2\left(\frac{\sqrt{3}}{2}\right) R=1.73 R$ from the worst case location located in the cell $C-0$. The distance of the interfering cell 


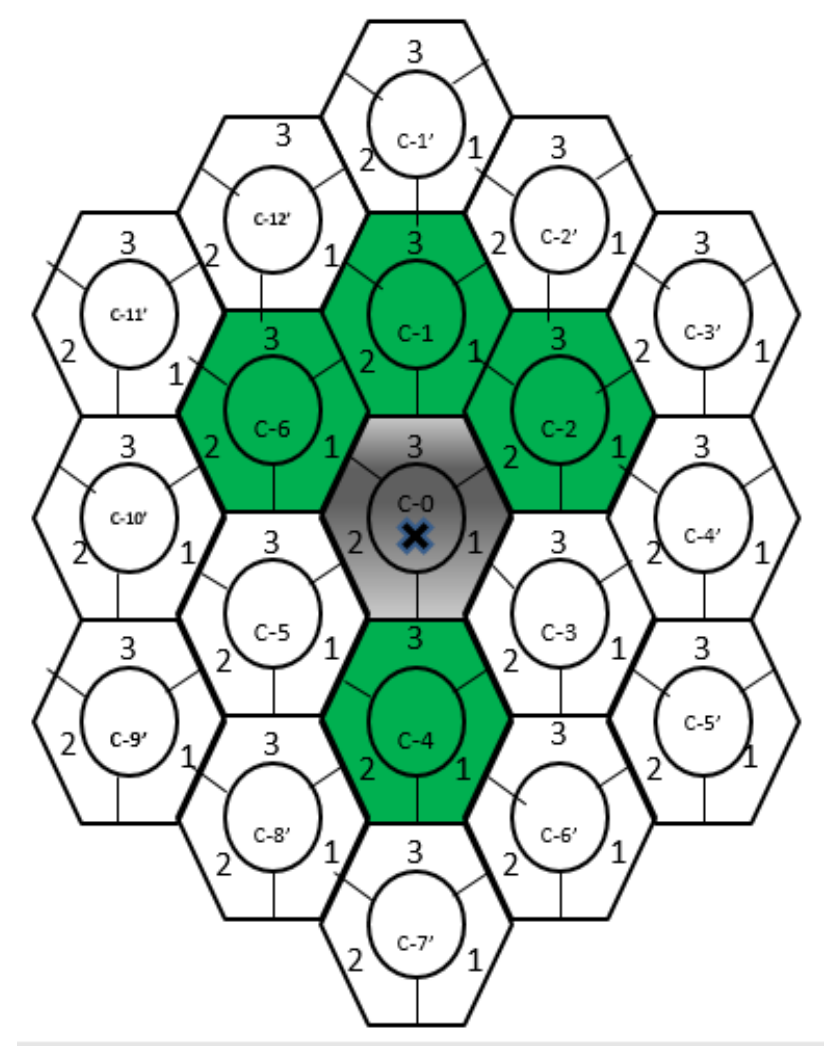

Figure 2.6: Mode No.5

$C-1^{\prime}$ is calculated as

$$
2 \sqrt{3} R \approx 3.46 R
$$

The distance of interfering cells $C-2^{\prime}, C-4^{\prime}, C-6^{\prime}, C-8^{\prime}, C-10^{\prime}$ and $C-12^{\prime}$ each are $3 R$ and are tabulated in Table 2.6.

In Mode No.7 shown in Fig. 2.8. the base stations taking part in useful signal transmission are $C-1, C-4$ and $C-6$. Cells $C-1$ and $C-4$ each are located at a distance of $2 R$ from the worst case location within cell $C-0$ while the distance of base station represented by $C-6$ and the interfering base station represented by $C-5$ is calculated as

$$
\sqrt{(2.5 R)^{2}+\left(\frac{\sqrt{3}}{2} R\right)^{2}} \approx 2.64 R
$$




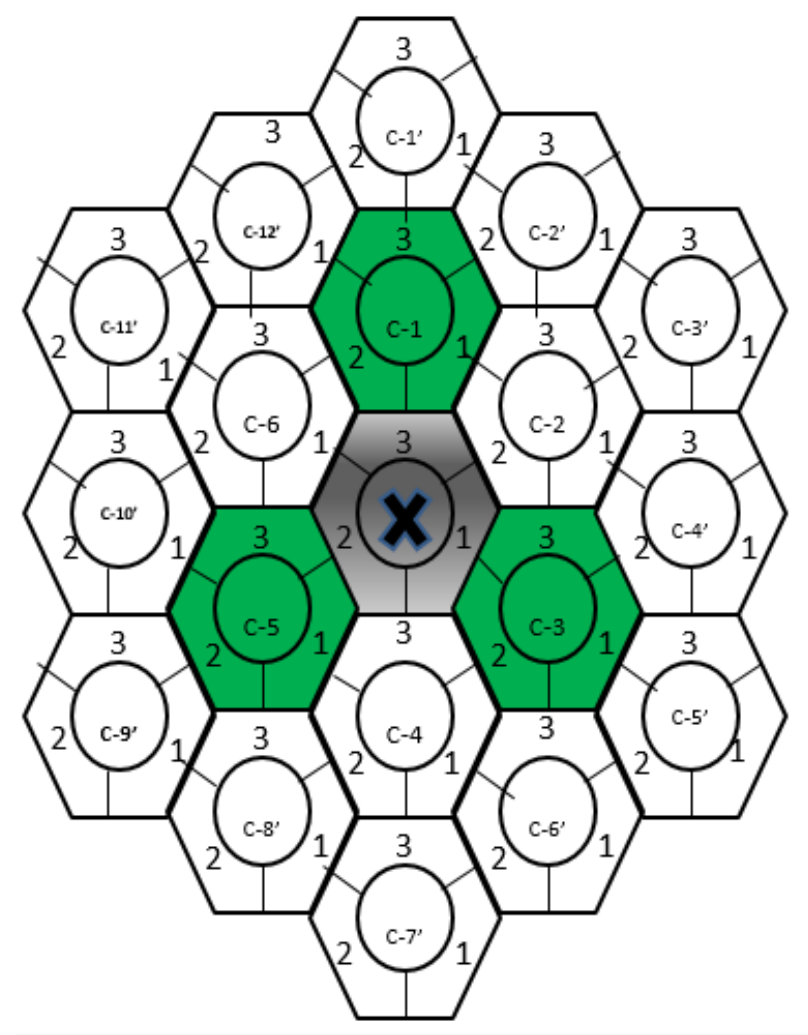

Figure 2.7: Mode No.6

The intefering cells in Mode No.7 are $C-1^{\prime}, C-9^{\prime}, C-10^{\prime}, C-11^{\prime}$ and $C-12^{\prime}$. In case of interfering cell $C-1^{\prime}$, the distance of the worst case location in Mode No.7 is

$$
\sqrt{(R)^{2}+\left(4 \cdot \frac{\sqrt{3}}{2} R\right)^{2}} \approx 3.60 R
$$

Similarly the distances from the other interfering cells are calculated and tabulated in Table 2.7.

In Mode No.8, base stations represented by cells $C-1, C-2$ and $C-6$ are taking part in useful transmission to users located in cell $C-0$ and user located at the worst case location of $C-0$. The interfering cells in this mode are $C-5, C-1^{\prime}, C-9^{\prime}, C-10^{\prime}, C-11^{\prime}$ and 
Table 2.6: Worst Case Location Distances in Mode No. 6

\begin{tabular}{|c|c|c|c|}
\hline $\begin{array}{l}\mathrm{BS} \\
(\mathrm{CoMP})\end{array}$ & $\begin{array}{l}\text { Worst Case Location } \\
\text { Distance }\end{array}$ & Interferers & $\begin{array}{l}\text { Worst Case Location } \\
\text { Distance }\end{array}$ \\
\hline $\begin{array}{l}C-1 \\
C-3 \\
C-5\end{array}$ & $\begin{array}{l}1.73 R \\
1.73 R \\
1.73 R\end{array}$ & $\begin{array}{l}C-2 \\
C-4 \\
C-6 \\
C-1^{\prime} \\
C-2^{\prime} \\
C-3^{\prime} \\
C-4^{\prime} \\
C-5^{\prime} \\
C-6^{\prime} \\
C-7^{\prime} \\
C-8^{\prime} \\
C-9^{\prime} \\
C-10^{\prime} \\
C-11^{\prime} \\
C-12^{\prime}\end{array}$ & $\begin{array}{l}1.73 R \\
1.73 R \\
1.73 R \\
3.46 R \\
3.00 R \\
3.46 R \\
3.00 R \\
3.46 R \\
3.00 R \\
3.46 R \\
3.00 R \\
3.46 R \\
3.00 R \\
3.46 R \\
3.00 R\end{array}$ \\
\hline
\end{tabular}

Table 2.7: Worst Case Location Distances in Mode No. 7

\begin{tabular}{|l|l|l|l|}
\hline \hline $\begin{array}{l}\text { BS } \\
(\mathrm{CoMP})\end{array}$ & $\begin{array}{l}\text { Worst Case Location } \\
\text { Distance }\end{array}$ & Interferers & $\begin{array}{l}\text { Worst Case Location } \\
\text { Distance }\end{array}$ \\
\hline$C-1$ & $2.00 R$ & $C-5$ & $2.64 R$ \\
$C-4$ & $2.00 R$ & $C-1^{\prime}$ & $3.60 R$ \\
$C-6$ & $2.64 R$ & $C-9^{\prime}$ & $4.36 R$ \\
& & $C-10^{\prime}$ & $4.00 R$ \\
& & $C-11^{\prime}$ & $4.36 R$ \\
& & $C-12^{\prime}$ & $3.60 R$ \\
\hline
\end{tabular}

$C-12^{\prime}$. The worst case location distance from each of the two cells $C-2$ and $C-6$ is

$$
\sqrt{(1.5 R)^{2}+\left(2 \cdot \frac{\sqrt{3}}{2} R\right)^{2}} \approx 2.29 R
$$

In Mode No.9 shown in Fig. 2.10, two worst case locations are possible having a distance of $2 R$ from the base stations represented by $C-1$ and $C-4$. Considering the worst case 


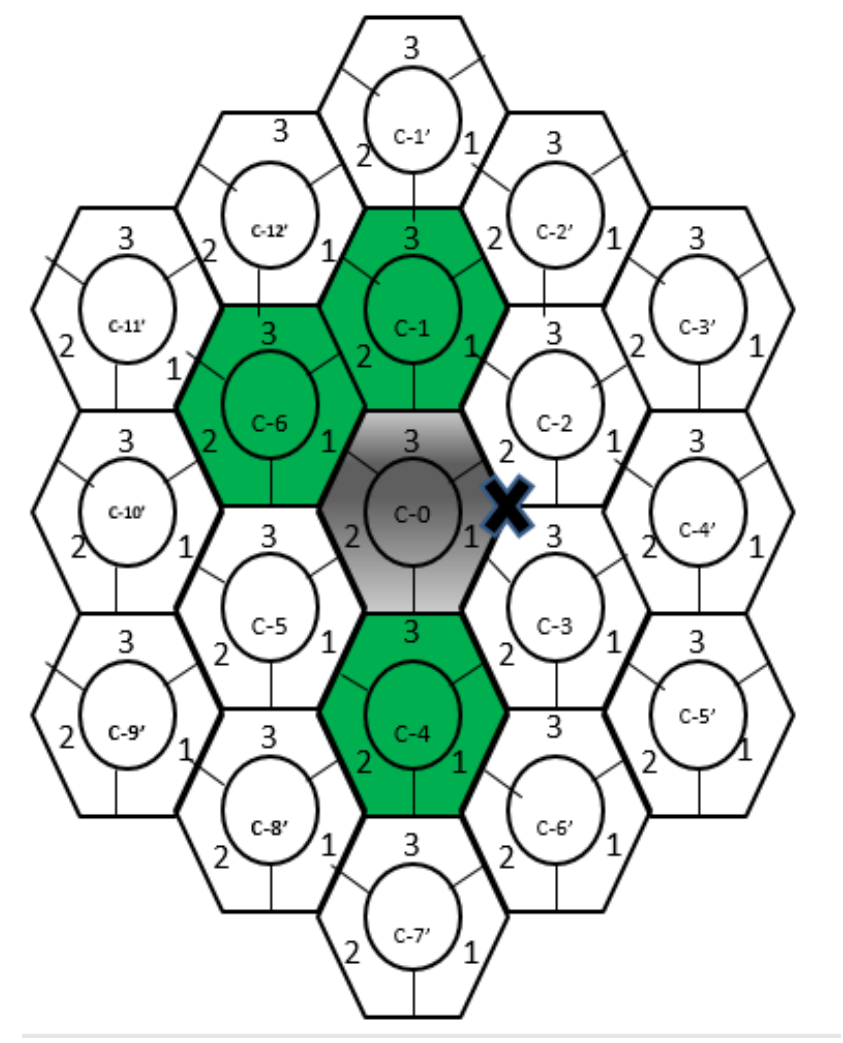

Figure 2.8: Mode No.7

Table 2.8: Worst Case Location Distances in Mode No. 8

\begin{tabular}{|l|l|l|l|}
\hline \hline BS & Worst Case Location & Interferers & $\begin{array}{l}\text { Worst Case Location } \\
\text { Distance }\end{array}$ \\
\hline$C-1$ & Distance & & $1.50 R$ \\
$C-2$ & $2.60 R$ & $C-5$ & $4.33 R$ \\
$C-6$ & $2.29 R$ & $C-1^{\prime}$ & $3.12 R$ \\
& $2.29 R$ & $C-9^{\prime}$ & $3.12 R$ \\
& & $C-10^{\prime}$ & $3.96 R$ \\
& & $C-11^{\prime}$ & $3.77 R$ \\
\hline
\end{tabular}

location on left side in cell $C-0$, the interfering cells in this mode are $C-2, C-3, C-1^{\prime}$, $C-2^{\prime}, C-3^{\prime}, C-4^{\prime}$, and $C-5^{\prime}$.

Similarly in the other modes the base stations taking part in CoMP transmission and the interfering cells are mentioned in the corresponding tables for each mode. 


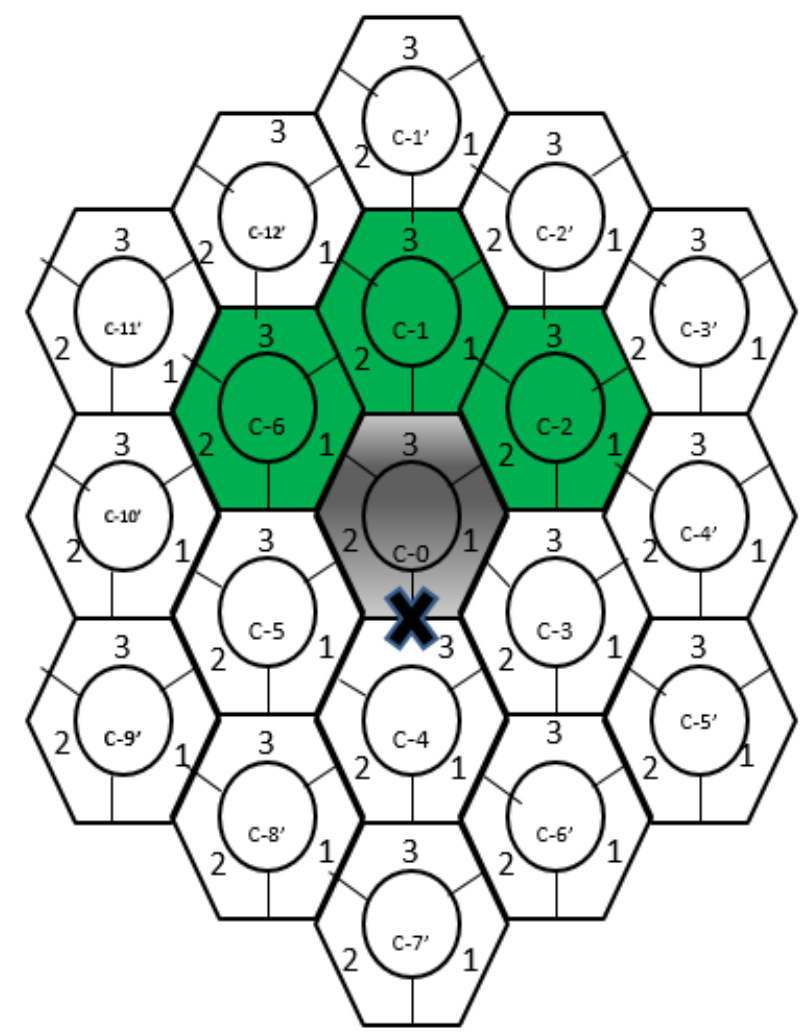

Figure 2.9: Mode No.8

Table 2.9: Worst Case Location Distances in Mode No. 9

\begin{tabular}{|l|l|l|l|}
\hline \hline BS & Worst Case Location & Interferers & $\begin{array}{l}\text { Worst Case Location } \\
\text { Distance }\end{array}$ \\
\hline$C-1$ & Distance & & $2.64 R$ \\
$C-4$ & $2 R$ & $C-2$ & $2.64 R$ \\
& $2 R$ & $C-3$ & $3.60 R$ \\
& & $C-1^{\prime}$ & $3.60 R$ \\
& & $C-2^{\prime}$ & $4.36 R$ \\
& & $C-3^{\prime}$ & $4.00 R$ \\
& & $C-4^{\prime}$ & $C-5^{\prime}$ \\
\hline
\end{tabular}

According to [21] the probability density function (PDF) of the random distance inside 


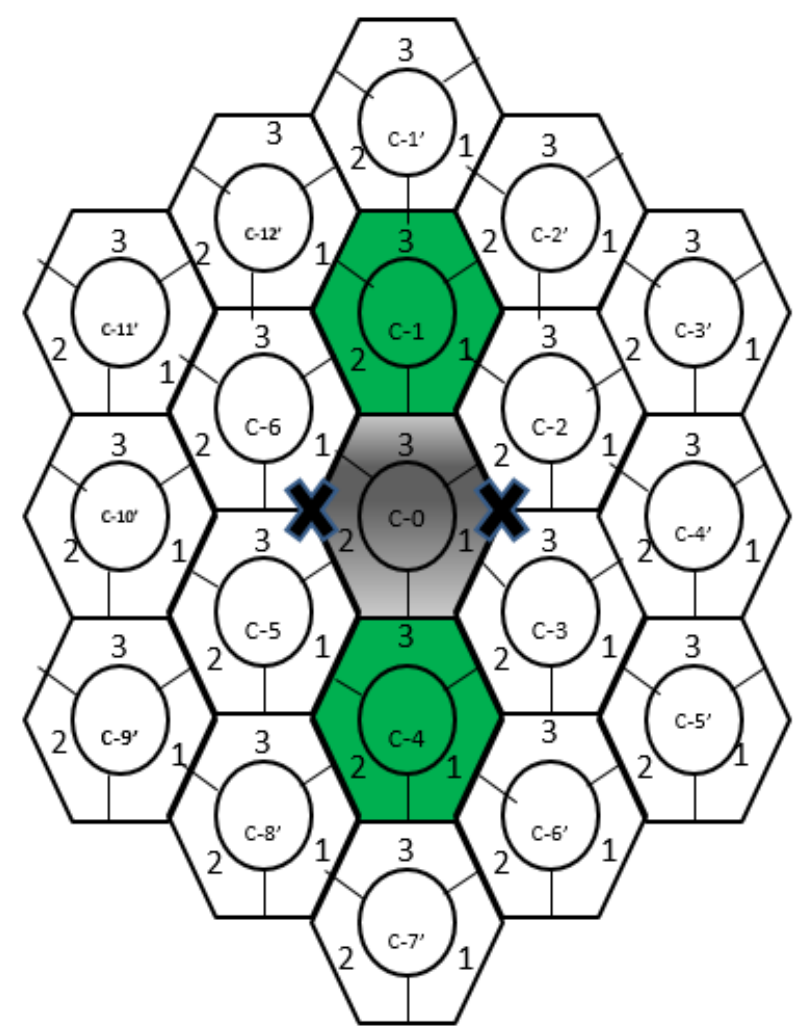

Figure 2.10: Mode No.9

Table 2.10: Worst Case Location Distances in Mode No. 10

\begin{tabular}{|l|l|l|l|}
\hline \hline BS & Worst Case Location & Interferers & $\begin{array}{l}\text { Worst Case Location } \\
\text { Distance }\end{array}$ \\
\hline$C-2$ & Distance & & $2.59 R$ \\
$C-6$ & $2.29 R$ & $C-1$ & $1.50 R$ \\
& $2.29 R$ & $C-5$ & $4.33 R$ \\
& & $C-1^{\prime}$ & $3.12 R$ \\
& & $C-9^{\prime}$ & $3.16 R$ \\
& & $C-10^{\prime}$ & $3.96 R$ \\
& & $C-11^{\prime}$ & $3.77 R$ \\
\hline
\end{tabular}

a hexagon is

$$
f_{D_{\mathrm{I}}}(x)=\frac{4 x}{\sqrt{3}} \begin{cases}\frac{\pi}{3} & 0 \leq x \leq \frac{\sqrt{3}}{2} \\ \frac{\pi}{3}-2 \cos ^{-1} \frac{\sqrt{3}}{2 x} & \frac{\sqrt{3}}{2} \leq x \leq 1 \\ 0 & \text { otherwise }\end{cases}
$$




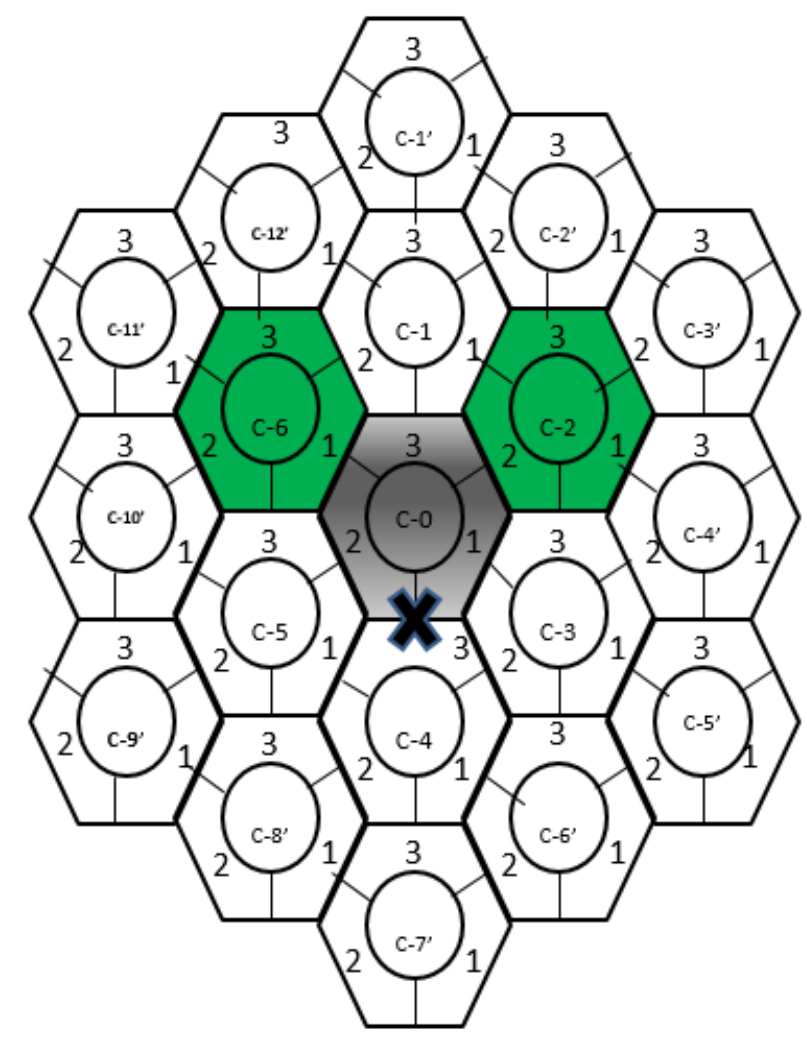

Figure 2.11: Mode No.10

Table 2.11: Worst Case Location Distances in Mode No. 11

\begin{tabular}{|l|l|l|l|}
\hline \hline BS & Worst Case Location & Interferers & $\begin{array}{l}\text { Worst Case Location } \\
\text { Distance }\end{array}$ \\
\hline$C-1$ & Distance & & $2.00 R$ \\
$C-2$ & $2.64 R$ & $C-3$ & $4.35 R$ \\
& $2.64 R$ & $C-1^{\prime}$ & $C .00 R$ \\
& & $C-2^{\prime}$ & $C-3^{\prime}$ \\
& & $C-4^{\prime}$ & $3.60 R$ \\
& & $C-5^{\prime}$ & $3.60 R$ \\
\hline
\end{tabular}

According to [21] PDF of random distances, from the center of one hexagon to an arbitrary 


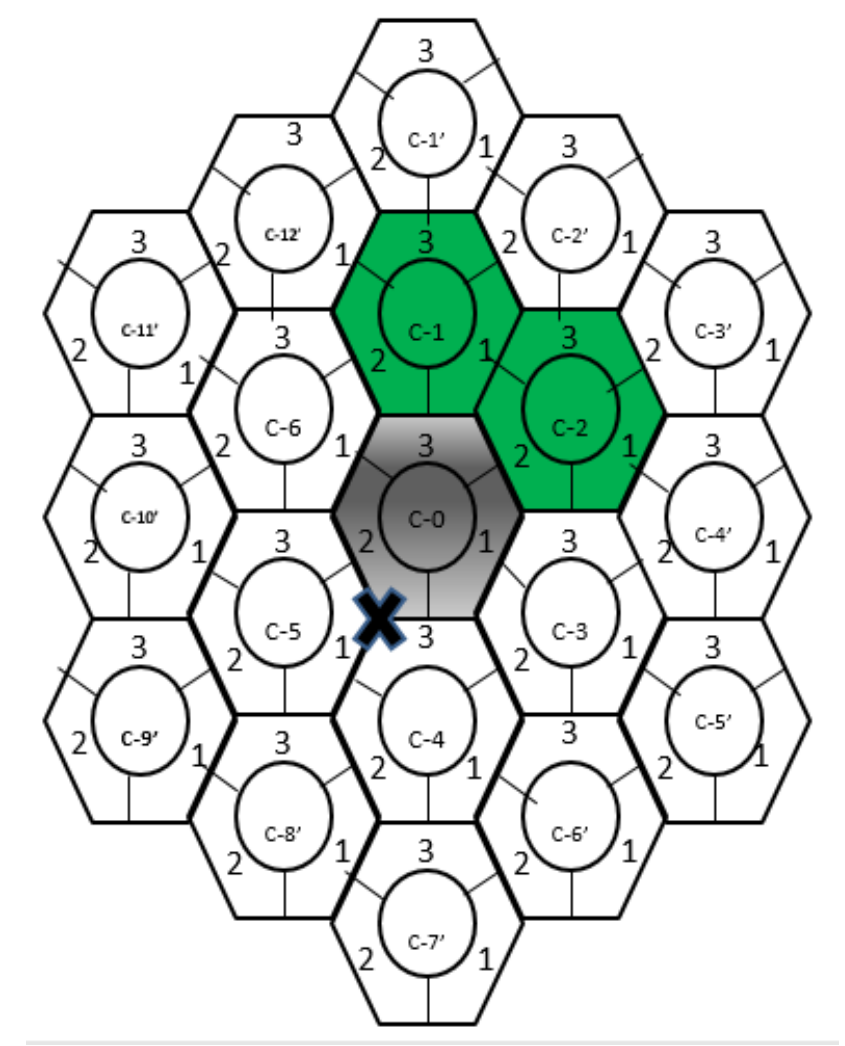

Figure 2.12: Mode No.11

Table 2.12: Worst Case Location Distances in Mode No. 12

\begin{tabular}{|l|l|l|l|}
\hline \hline BS \\
$(\mathrm{CoMP})$ & Worst Case Location & Interferers & $\begin{array}{l}\text { Worst Case Location } \\
\text { Distance }\end{array}$ \\
\hline$C-1$ & $2.64 R$ & $C-2$ & $2.64 R$ \\
& & $C-3$ & $2.00 R$ \\
& & $C-1^{\prime}$ & $4.36 R$ \\
& & $C-2^{\prime}$ & $4.00 R$ \\
& & $C-3^{\prime}$ & $4.36 R$ \\
& & $C-4^{\prime}$ & $3.60 R$ \\
& & $C-5^{\prime}$ & $3.60 R$ \\
\hline
\end{tabular}

node in an adjacent hexagon is

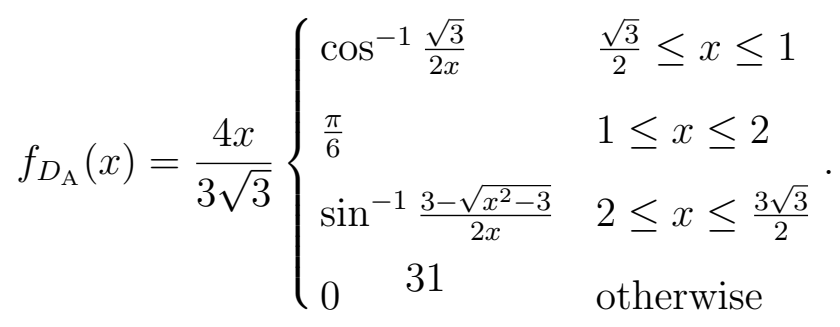




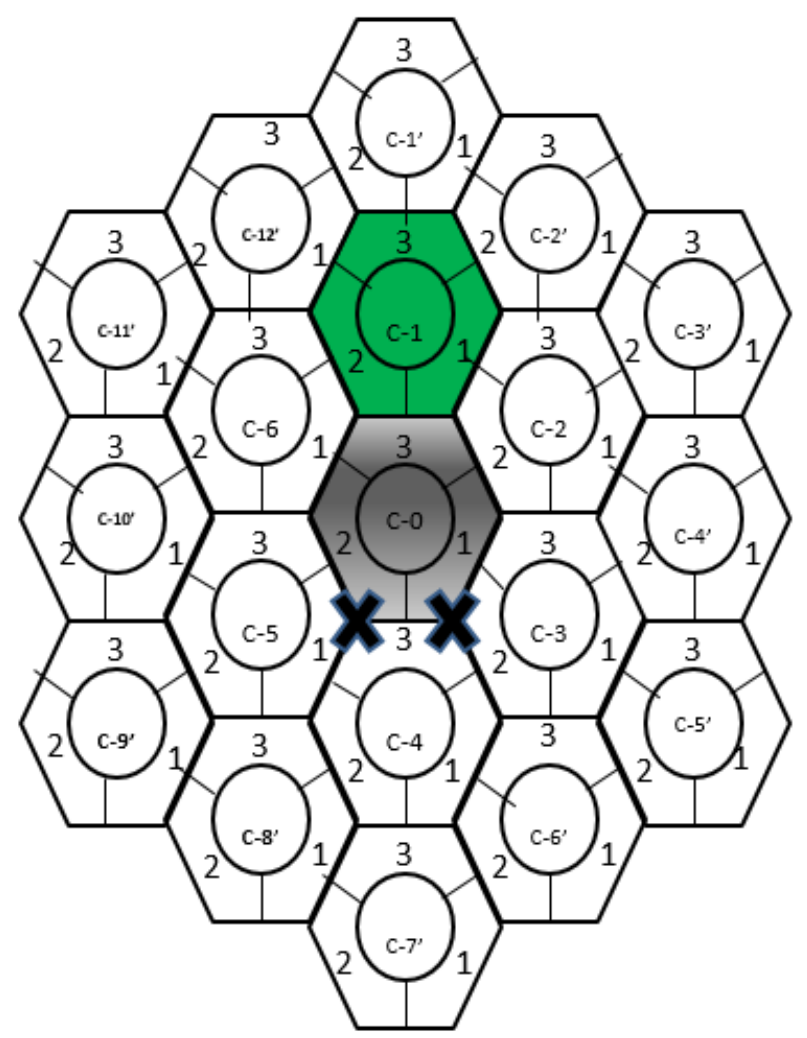

Figure 2.13: Mode No.12

Given the PDF, the expected value of SNR for Mode No.0 (i.e the conventional operation of cell $C-0$ without any CoMP transmission by neighbouring base stations in its coverage area) can be written as [21]

$$
E[S N R]_{M o d e-0}=\frac{P_{t x} \int_{0}^{1} x^{-\alpha} f_{D_{\mathrm{I}}}(x) d x}{N_{0}} .
$$

The above expression gives the expectation of SNR in case of downlink for user located in the central cell $C-0$. 
Taking all the limits into consideration, Eq. (2.19) is further expanded as

$$
E[S N R]_{M o d e-0}=\frac{\frac{4 \pi}{3 \sqrt{3}} P_{t x} \int_{0}^{\frac{\sqrt{3}}{2}} x^{1-\alpha} d x+\frac{4 \pi}{3 \sqrt{3}} P_{t x} \int_{\frac{\sqrt{3}}{2}}^{1} x^{1-\alpha} d x-\frac{8}{\sqrt{3}} P_{t x} \int_{\frac{\sqrt{3}}{2}}^{1} x^{1-\alpha} \cos ^{-1} \frac{\sqrt{3}}{2 x} d x}{N_{0}} .
$$

According to [22] the channel coefficient $|z|^{2}=x^{-\alpha} S_{n}\left|F_{n}\right|^{2}$, whereas $\alpha$ is the path loss exponent, $S_{n}$ is the shadowing power gain and $F_{n}$ is the complex microscopic fading component, $S_{n}$ and $F_{n}$ are independent and are also independent of link distance $x$. Further it is assumed that the mean gain from shadowing and microscopic fading are normalized to unity i.e $E\left[S_{n}\right]=1$ and $E\left[\left|F_{n}\right|^{2}\right]=1$. According to [22] the mean SNR can be written as

$$
E[S N R]=\frac{P_{t x} \sum_{c b=1}^{N_{b}} \int_{\frac{\sqrt{3}}{2}}^{\frac{3 \sqrt{3}}{2}} x^{-\alpha} f_{D_{\mathrm{A}}}(x) d x}{N_{0}}
$$

In Mode No.1 where six base stations are taking part in CoMP transmission in the coverage area of the switched off cell $C-0$ Eq. (2.21) becomes as

$$
E[S N R]_{M o d e-1}=\frac{\sum_{c b=1}^{6} P_{t x}\left\{\int_{\frac{\sqrt{3}}{2}}^{1} x^{-\alpha} f_{D_{\mathrm{A}}} d x+\int_{1}^{2} x^{-\alpha} f_{D_{\mathrm{A}}} d x+\int_{2}^{\frac{3 \sqrt{3}}{2}} x^{-\alpha} f_{D_{\mathrm{A}}} d x\right\}}{N_{0}}
$$

Considering $\alpha=4$ and putting the values of $f_{D_{\mathrm{A}}}(x)$ from Eq. (2.18) in Eq. (2.22) we get

$E[S N R]_{\text {Mode-1 }}=\frac{\sum_{c b=1}^{6} P_{t x} \frac{4}{3 \sqrt{3}}\left\{\int_{\frac{\sqrt{3}}{2}}^{1} x^{-3} \cos ^{-1} \frac{\sqrt{3}}{2 x} d x+\int_{1}^{2} x^{-3} \frac{\pi}{6} d x+\int_{2}^{\frac{3 \sqrt{3}}{2}} x^{-3} \sin ^{-1} \frac{3-\sqrt{x^{2}-3}}{2 x} d x\right\}}{N_{0}}$. 
For other modes we have

$E[S N R]_{M o d e-2}=\frac{\sum_{c b=1}^{5} P_{t x} \frac{4}{3 \sqrt{3}}\left\{\int_{\frac{\sqrt{3}}{2}}^{1} x^{-3} \cos ^{-1} \frac{\sqrt{3}}{2 x} d x+\int_{1}^{2} x^{-3} \frac{\pi}{6} d x+\int_{2}^{\frac{3 \sqrt{3}}{2}} x^{-3} \sin ^{-1} \frac{3-\sqrt{x^{2}-3}}{2 x} d x\right\}}{N_{0}}$.

$E[S N R]_{M o d e-3}=\frac{\sum_{c b=1}^{4} P_{t x} \frac{4}{3 \sqrt{3}}\left\{\int_{\frac{\sqrt{3}}{2}}^{1} x^{-3} \cos ^{-1} \frac{\sqrt{3}}{2 x} d x+\int_{1}^{2} x^{-3} \frac{\pi}{6} d x+\int_{2}^{\frac{3 \sqrt{3}}{2}} x^{-3} \sin ^{-1} \frac{3-\sqrt{x^{2}-3}}{2 x} d x\right\}}{N_{0}}$.

$E[S N R]_{M o d e-4}=\frac{\sum_{c b=1}^{4} P_{t x} \frac{4}{3 \sqrt{3}}\left\{\int_{\frac{\sqrt{3}}{2}}^{1} x^{-3} \cos ^{-1} \frac{\sqrt{3}}{2 x} d x+\int_{1}^{2} x^{-3} \frac{\pi}{6} d x+\int_{2}^{\frac{3 \sqrt{3}}{2}} x^{-3} \sin ^{-1} \frac{3-\sqrt{x^{2}-3}}{2 x} d x\right\}}{N_{0}}$.

Similarly for other Modes $E[S N R]$ can be expressed. In Mode No.7 three base stations are taking part in CoMP transmission and the expected value of SNR is given as

$E[S N R]_{M o d e-7}=\frac{\sum_{c b=1}^{3} P_{t x} \frac{4}{3 \sqrt{3}}\left\{\int_{\frac{\sqrt{3}}{2}}^{1} x^{-3} \cos ^{-1} \frac{\sqrt{3}}{2 x} d x+\int_{1}^{2} x^{-3} \frac{\pi}{6} d x+\int_{2}^{\frac{3 \sqrt{3}}{2}} x^{-3} \sin ^{-1} \frac{3-\sqrt{x^{2}-3}}{2 x} d x\right\}}{N_{0}}$.

In Mode No.12 as shown in Fig. 2.13, two worst case locations are possible. The expected value of SNR in Mode No.12 is given as

$E[S N R]_{M o d e-12}=\frac{P_{t x} \frac{4}{3 \sqrt{3}}\left\{\int_{\frac{\sqrt{3}}{2}}^{1} x^{-3} \cos ^{-1} \frac{\sqrt{3}}{2 x} d x+\int_{1}^{2} x^{-3} \frac{\pi}{6} d x+\int_{2}^{\frac{3 \sqrt{3}}{2}} x^{-3} \sin ^{-1} \frac{3-\sqrt{x^{2}-3}}{2 x} d x\right\}}{N_{0}}$.

The per user capacity based on the expected value of SNR within a cell can then be calculated for each switching mode using Shannon formula $C=W \log _{2}(1+\gamma)$, whereas $\gamma=E[S N R]$. The closed form expressions of the three integrals mentioned in Eq. (2.23) to (2.28) for 
finding out $\mathrm{E}[\mathrm{SNR}]$ of switching modes is given as,

$$
\begin{gathered}
I_{1}=\int x^{-3} \cos ^{-1} \frac{\sqrt{3}}{2 x} d x=-\frac{t^{2}}{2} \cos ^{-1} \frac{\sqrt{3}}{2} t-\frac{1}{3} \sin ^{-1} \frac{\sqrt{3}}{2} t+\frac{1}{4} t \sqrt{\frac{4}{3}-t^{2}} \\
I_{2}=\int x^{-3} \frac{\pi}{6} d x=-\frac{\pi}{12} t^{-2} .
\end{gathered}
$$

whereas $x^{-1}=t$ and $x^{-2} d x=-d t$.

$$
I_{3}=\int x^{-3} \sin ^{-1} \frac{3-\sqrt{x^{2}-3}}{2 x} d x=\frac{1}{6}\left\{-\frac{\cos 2 \theta}{2} \sin ^{-1}\left(\frac{\sqrt{3}}{2} \cos \theta-\frac{\sin \theta}{2}\right)-\frac{1}{8} \sin 2 \theta\right\}
$$

whereas $x=\sqrt{3} \sec \theta$ and $d x=\sqrt{3} \sec \theta \tan \theta d \theta$ whereas $\sec \theta=\frac{1}{\cos \theta}$.

A step by step integration of $I_{1}, I_{2}$ and $I_{3}$ is given in Appendix B. 


\section{Chapter 3}

\section{Power Consumption}

\subsection{Introduction}

The performance of a cellular network can be evaluated through energy consumption ratio (ECR) in Joules per bit which is a ratio between energy consumption and capacity and the reciprocal of ECR is the Energy Efficiency Ratio (EER) in bits per Joule [23]. In order to find out the EER in this work it is necessary to find out the energy consumption at a base station site.

In the subsequent section only the operational energy consumption of a base station site is considered and the embodied energy which is the energy consumed by all processes associated with the production of base station site equipments is not taken into consideration.

According to [6] and as shown in Fig. 3.1, the power consumption of a UMTS BS is more during peak traffic hours. This thesis is considering the off-peak traffic periods and during low traffic periods the effect of traffic on BS power consumption is considered to be negligible.

Power consumption models for the base station of the access network of a cellular network have been presented in different papers [24],[25],[26] and [27]. But the power consumption 


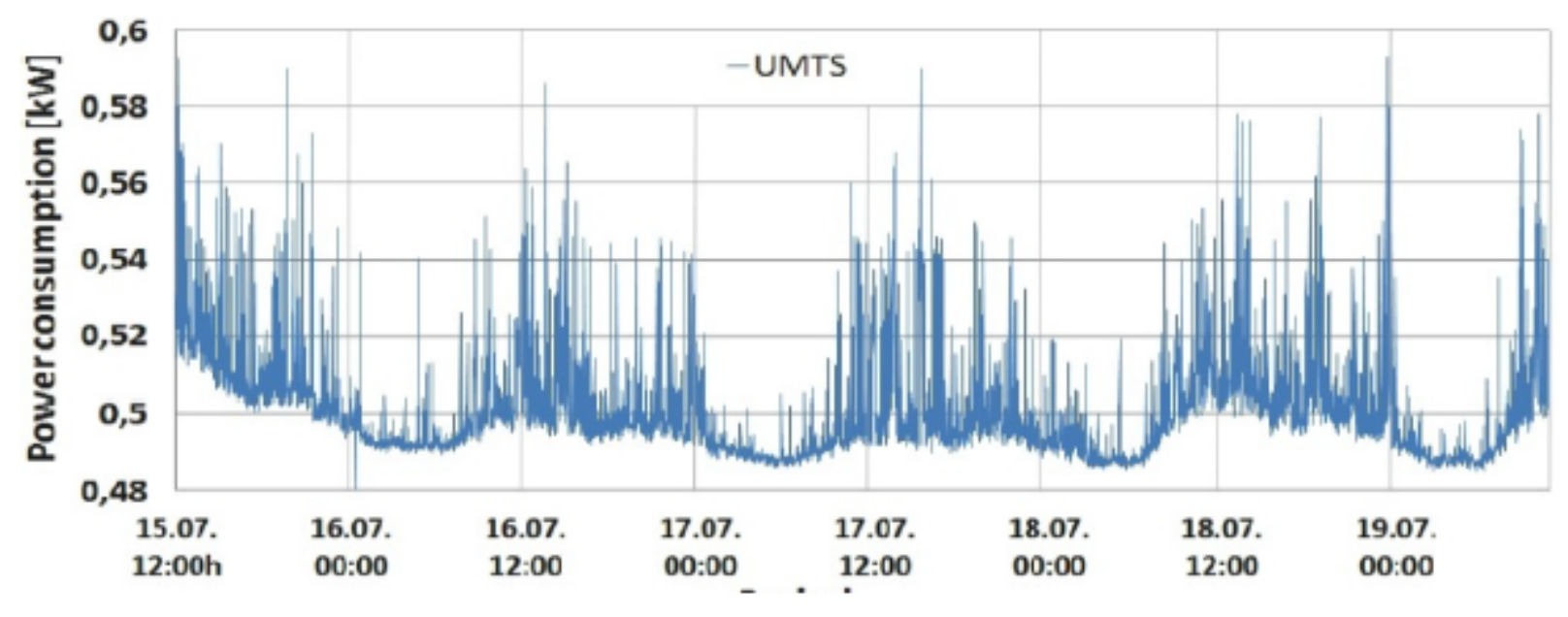

Figure 3.1: Power Consumption of UMTS BS [6]

models presented in those papers have not taken into account the increase in power consumption due to the usage of CoMP transmission which has been included in LTE-Advanced standard. In other words the power consumption models available in literature are for conventional stand alone operation of BS without taking CoMP into account. In the following section a power consumption model of base station site taking part in CoMP transmission is developed.

\subsection{Power Consumption Model}

Consider a cellular base station site which consists of a base station $B$, air conditioner unit, battery bank, rectifier, backhaul transmission system (i.e microwave transmission system), alarm systems (humidity, temperature, fire, door alarm etc.), and site lighting.

The base station consists of two main subsystems, i.e, Signal Processing Subsystem or Signal Processing Unit (SPU) and the Radio Resource Subsystem or Radio Resource Unit (RRU). The Radio Resource Subsystem is composed of the High Power Amplifiers (HPAs), Tranciever Modules (TRXs), Low Noise Amplifiers (LNAs) and internal cooling fans. 
The power consumption at base station site $P_{B S-\text { site }}$ is composed of the power consumption of the air conditioning system $P_{\text {air.con }}$, the input power to the rectifier $P_{\text {rect-in }}$, the power consumption due to backhauling $P_{\text {backhauling }}$, power consumption in dc/dc conversion and due to site lighting and other alarm systems represented by $P_{\text {Misc }}$ as shown in Fig. 3.2 and is expressed as

$$
P_{B S-\text { site }}=P_{\text {rect-in }}+P_{\text {air.con }}+P_{\text {backhauling }}+P_{\text {Misc }} .
$$

The heat generated by BS at the site is removed by air conditioning unit having power consumption $P_{\text {air.con }}$ in Eq. (3.1). The heat generated by backhauling, alarm and monitoring system is assumed to be negligible as compared to the heat generated by the base station hardware.

The rectifier converts the $110 \mathrm{~V} / 220 \mathrm{~V}$ commercial ac power into $-48 \mathrm{~V}$ dc power, which then is responsible for charging of battery bank and fed as an input to the DC/DC converter which converts $-48 \mathrm{~V}$ dc into lower dc voltages required by base station modules. During the rectification and power conversion process some electrical energy is converted into heat and is lost.

Let $\mu_{\text {rect }}$ be the efficiency of the rectifier. The rectifier efficiency $\mu_{\text {rect }}$ is give as

$$
\begin{gathered}
\mu_{\text {rect }}=\frac{P_{\text {rect-out }}}{P_{\text {rect-in }}}, \\
P_{\text {rect-in }}=\frac{P_{\text {rect-out }}}{\mu_{\text {rect }}} .
\end{gathered}
$$

The rectifier output power $P_{\text {rect-out }}$ is used for battery backup charging and is also fed as input to DC/DC convertor. Therefore Eq. (3.2) becomes as

$$
P_{\text {rect-in }}=\frac{P_{d c / d c_{i n}}+P_{\text {battery-backup }}}{\mu_{\text {rect }}} .
$$




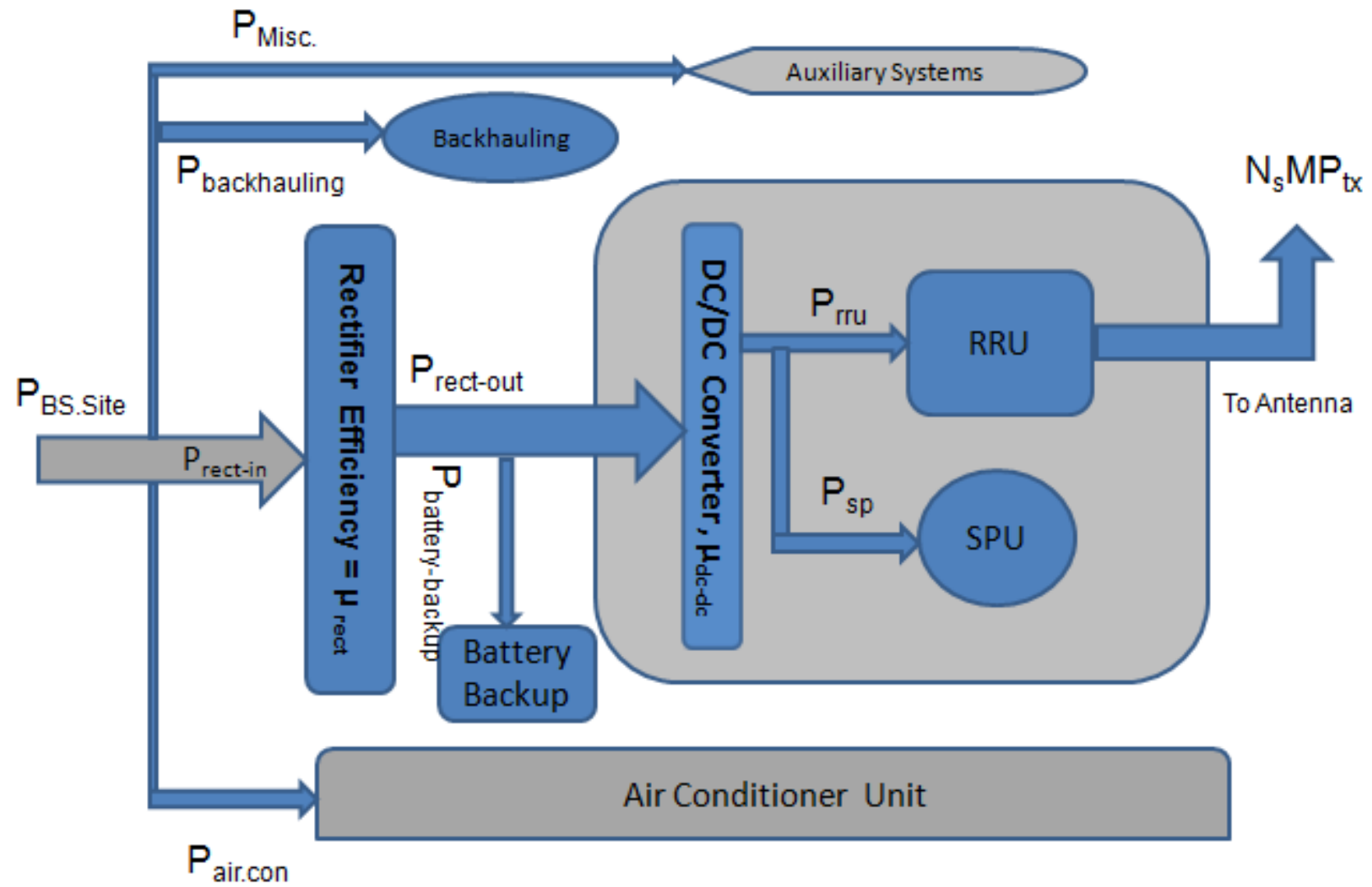

Figure 3.2: Power Consumption of a BS Site 
Let a fraction $\beta_{0}$ times of rectifier output power $P_{\text {rect-out }}$ is used for battery backup charging only in case it is discharged due to the unavailability of commercial A.C power or due to failure of diesel generator set. The remaining fraction $\left(1-\beta_{0}\right) P_{\text {rect-out }}$ is fed as input to the $\mathrm{DC} / \mathrm{DC}$ convertor and is represented as $P_{d c-d c_{i n}}$.

$$
P_{\text {battery-backup }}=\beta_{0} P_{\text {rect-out }}=\beta_{0} \mu_{\text {rect }} P_{\text {rect-in }}
$$

Let the efficiency of DC/DC convertor be $\mu_{d c-d c}$.

$$
P_{d c-d c_{i n}}=\frac{P_{d c-d c_{o u t}}}{\mu_{d c-d c}}
$$

The DC/DC converter output power $P_{d c-d c_{o u t}}$ is used for the operation of Signal Processing Unit (Main Unit) and the Radio Resource Unit, the corresponding powers are represented by $P_{s p}$ and $P_{r r u}$ and Eq. (3.4) becomes as

$$
P_{d c-d c_{i n}}=\frac{P_{s p}+P_{r r u}}{\mu_{d c-d c}} .
$$

Let $\beta_{1}$ fraction of $\mathrm{DC} / \mathrm{DC}$ convertor output power $P_{d c-d c_{\text {out }}}$ is used for Radio Resource Unit i.e $\beta_{1} P_{d c-d c_{\text {out }}}=P_{\text {rru }}$ then Eq. (3.5) becomes as

$$
P_{d c-d c_{i n}}=\frac{P_{s p}+\beta_{1} P_{d c-d c_{o u t}}}{\mu_{d c-d c}}
$$

Putting value of $P_{d c-d c_{i n}}$ from Eq. (3.4) into Eq. (3.6) we have

$$
\begin{aligned}
& \frac{P_{d c-d c_{o u t}}}{\mu_{d c-d c}}=\frac{P_{s p}}{\mu_{d c-d c}}+\beta_{1} \frac{P_{d c-d c_{o u t}}}{\mu_{d c-d c}}, \\
& \frac{P_{d c-d c_{o u t}}}{\mu_{d c-d c}}-\beta_{1} \frac{P_{d c-d c_{o u t}}}{\mu_{d c-d c}}=\frac{P_{s p}}{\mu_{d c-d c}},
\end{aligned}
$$




$$
\begin{gathered}
\frac{P_{d c-d c_{o u t}}\left(1-\beta_{1}\right)}{\mu_{d c-d c}}=\frac{P_{s p}}{\mu_{d c-d c}}, \\
\frac{P_{d c-d c_{o u t}}\left(1-\beta_{1}\right)\left(P_{d c-d c_{\text {in }}}\right)}{\left(P_{d c-d c_{\text {out }}}\right)}=\frac{P_{s p}}{\mu_{d c-d c}}, \\
P_{d c-d c_{\text {in }}}\left(1-\beta_{1}\right)=\frac{P_{s p}}{\mu_{d c-d c}} .
\end{gathered}
$$

Putting $P_{d c-d c_{i n}}=\left(1-\beta_{0}\right) P_{\text {rect-out }}$ in Eq.(3.7) we get

$$
\begin{gathered}
\left(1-\beta_{0}\right) P_{\text {rect-out }}\left(1-\beta_{1}\right)=\frac{P_{s p}}{\mu_{d c-d c}}, \\
\left(1-\beta_{0}\right)\left(1-\beta_{1}\right) \mu_{\text {rect }} P_{\text {rect-in }}=\frac{P_{s p}}{\mu_{d c-d c}}, \\
P_{\text {rect-in }}=\frac{P_{s p}}{\mu_{d c-d c}\left(1-\beta_{0}\right)\left(1-\beta_{1}\right) \mu_{r e c t}} .
\end{gathered}
$$

According to [28] $P_{s p}$ has a base value of $58 \mathrm{~W}$ and increase in $P_{s p}$ per sector due to CoMP is given as

$$
P_{s p-C o M P}=58\left(0.87+0.1 N_{c}+0.03 N_{c}^{2}\right)
$$

whereas $N_{c}$ is the cooperating set degree. The possible value for $N_{c}$ are $N_{c}>=2$ when base stations are taking part in CoMP transmission. The value of $N_{c}$ is 1 when there is no cooperation of base stations.

Taking into consideration power consumption due to cooperation among base stations Eq. (3.8) becomes as

$$
P_{\text {rect-in }}=N_{s} \frac{58\left(0.87+0.1 N_{C}+0.03 N_{C}^{2}\right)}{\mu_{d c-d c}\left(1-\beta_{0}\right)\left(1-\beta_{1}\right) \mu_{\text {rect }}} .
$$

Application of CoMP requires exchange of information among all base stations involved in CoMP transmission. This additional communication due to CoMP transmission between base stations shall result in increase of energy consumption in backhauling [28] and is given 
as

$$
P_{\text {backhauling-CoMP }}=\frac{C_{\text {backhauling }}}{100 \text { Mbits } / \text { sec }} 50 \mathrm{~W},
$$

whereas $C_{\text {backhauling }}$ is given as

$$
C_{\text {backhauling }}=\frac{N_{c}\left(2 N_{C}\right) p \cdot q}{T_{S}} \text { bits } / \text { sec }
$$

whereas $p$ is the average pilot density in time and frequency and $q$ is amount of feed back bits employed to feed back channel state information (CSI) of acceptable quality. $T_{s}$ is the symbol period having value $66.7 \mu$ sec.

For value of $N_{c}=1, P_{\text {backhauling-CoMP }}=0$.

To determine $P_{\text {air.cond }}$ i.e the power consumed by the air conditioner unit, let $\eta$ be the energy efficiency rating of the air conditioner unit.

Energy efficiency rating of air conditioner unit in BTU/Watts shows how many BTU per hour are removed for each watt of power that air conditioner unit draws. The difference between the power input to the rectifier and $N_{s} M P_{t x} \omega$ i.e the power fed to the antenna (assuming losses in antenna feeder cable to be negligible) is consumed and is converted into heat, whereas $\omega$ is antenna system efficiency, $M$ represents utilization of MIMO and $N_{s}$ represents number of sectors.

To remove that heat from the base station site the power consumed by the air conditioner unit is given as

$$
P_{\text {air.cond }}=\left[\frac{58 N_{s}\left(0.87+0.1 N_{C}+0.03 N_{C}^{2}\right)}{\mu_{d c-d c}\left(1-\beta_{0}\right)\left(1-\beta_{1}\right) \mu_{\text {rect }}}-N_{s} M P_{t x} \omega\right](3.412 / \eta)
$$

whereas 1 watt of power converted into heat generates 3.412 BTU/hr. Therefore Eq. (3.1) 
becomes as

$$
P_{B S-\text { site }}=\frac{58 N_{s}\left(0.87+0.1 N_{C}+0.03 N_{C}^{2}\right)}{\mu_{d c-d c}\left(1-\beta_{0}\right)\left(1-\beta_{1}\right) \mu_{\text {rect }}}+P_{\text {air.cond }}+P_{\text {backhauling }}+P_{\text {Misc }}
$$

whereas $P_{\text {air.cond }}$ is given by Eq. (3.13) and $P_{\text {backhauling }}$ includes the increase in backhauling power consumption due to CoMP given by Eq. (3.11).

\subsection{Base Station Sectors Taking Part in CoMP Trans- mission in Each Switching Mode}

In this work it is assumed that each base station has three sectors i.e $N_{s}=3$, however in real networks there may be up to six sectors in a base station. Each sector consists of a transmitter, receiver, High Power Amplifier (HPA), Low Noise Amplifier (LNA) and separate sectorized antennas. Each sector has an associated cost with respect to energy consumption. Each neighbouring base station of the central base station $C-0$ taking part in CoMP transmission in the coverage area of base station $C-0$ has two types of energy consumptions i.e energy consumption without taking part in CoMP transmission to provide service in its own coverage area, and additional energy consumed in CoMP operation of base station to provide service in the coverage area of the switched off base station $C-0$. Therefore the net energy consumed by a neighbouring base station to provide service in the coverage area of base station $C-0$ is the difference of the two types of energy consumptions. The associated difference of power is represented by $\Delta P$ and is given as

$$
\Delta P=P_{(B S-\text { site-CoMP })}-P_{(B S-\text { site-Conven })},
$$


whereas $P_{(B S-\text { site-CoMP })}$ is $\mathrm{BS}$ site power consumption in CoMP mode and $P_{(B S-\text { site-Conven })}$ is the power consumption of BS in conventional mode of operation.

In switching Mode No.1 shown in Fig. 2.2, sectors 1,2 of base station $C-1$, sector 2 of base station $C-2$, sectors 2,3 of base station $C-3$, sector 3 of base station $C-4$, sectors 1,3 of base station $C-5$ and sector 1 of base station $C-6$ are taking part in CoMP transmission in the coverage area of the switched off base station $C-0$. All the other sectors of the neighbouring base stations $C-1, C-2, C-3, C-4, C-5$ and $C-6$ are taking part in transmission in their corresponding areas in the conventional manner.

Similarly in Mode No.7 sectors 1 and 2 of base station $C-1$, sector 3 of base station $C-4$ and sector 1 of base station $C-6$ are taking part in CoMP transmission in the coverage area of switched off base station $C-0$.

Similarly for all other switching modes the number of sectors taking part in CoMP transmission can be found. Each of these sectors have an associated power consumption and only the power required for CoMP transmission in the switched off cell is taken into account in final analysis of finding EER. 


\subsection{Analysis}

The power consumption model in the previous section shows that the energy consumption at any base station site taking part in CoMP transmission is dependent on the value of $N_{c}$, the set degree of the base station cooperation. A higher value of $N_{c}$ causes not only an increase in base station energy consumption but also effects the energy consumed by the air conditioning unit.

The increase in energy consumption of signal processing, rectifier, DC/DC converter and backhauling shall cause more heat to be generated at base station site causing more operational requirement by the air conditioning unit. As compared to the earlier work the efficiency of DC/DC convertor and the rectifier units have been segregated since rectifier and DC/DC converter are separate hardware units having different functions and different efficiency. As compared to earlier base station power consumption models which are applicable to the $2^{\text {nd }}$ and $3^{\text {rd }}$ generation of cellular networks, this model can be applied to $4 \mathrm{G}$ and latter cellular network standard.

With the help of this model, the energy cost of providing service in the switched off base station coverage area can be calculated during off-peak traffic period using CoMP transmission.

As discussed in the Introduction part of this chapter, the effect of traffic on the power consumption of a BS has not been taken into account in this work since this thesis is considering the low traffic periods only and the effect of low traffic on power consumption of a BS can be considered as negligible.

The bits per Joule which is equal to bits/Watts-sec = bits/Joule can be found for each of the switching mode in this work using the model presented in this chapter.

A comparison of energy required at a BS site for conventional operation of BS's (BS's remaining operational $24 / 7$ irrespective of traffic load) to that of switching off low traffic 
BS's and using CoMP transmission to cover the switched off area, can be done.

This comparison provides an insight into the cost associated with the CoMP usage. In this model a cluster of seven BS's have been considred and this work can be extended to the whole cellular network.

Once the amount of power that can be saved is known, the number of electricity units saved in kilowatt-hour $(\mathrm{kWh})$ can be computed. Besides the monetry cost of energy for the whole access network can be calculated. The associated equivalent amount of $\mathrm{CO}_{2}$ whose emission into the environment can be prevented, can be found out from the number of electricity units in $\mathrm{kWh}$ as discussed in next chapter. 


\section{Chapter 4}

\section{Numerical Results and Analysis}

In this chapter results based on the work of Chapter 2 and Chapter 3 are discussed. The power required to cover a particular hexagonal cell $C-0$ in conventional manner, and the power required by neighbouring BS's using CoMP transmission to provide service in the coverage area of $C-0$ when it is turned off in off-peak traffic hours are determined. The EER in both cases is found and compared.

The effect of degree of cooperation set $N_{c}$ on the required power for the BS operation in CoMP switching modes is found.

In this work another objective is to find out whether the cost of operation (energy consumption of cellular network) could be reduced or not by turning off some of the base stations which are experiencing very low traffic at times such as night and on holidays. At the same time the trouble free communication in the switched off base station coverage area needs to be ensured by the neighbouring base stations taking part in CoMP transmission and whether the QoS of a user as it was when the BS was operational in conventional manner can be ensured by CoMP operation of neighbouring BS's.

Besides, the subsystems responsible for most of the power required are identified in order to further set a direction for future research on reducing energy consumption of those 
particular subsystems.

System parameters used for results are shown in Table 4.1 and Table 4.2.

Table 4.1: System Parameters for the Worst Case Locations

\begin{tabular}{|l|l|}
\hline Parameter & Value Used \\
\hline Transmit Power $P_{t x}$ & 20 Watts \\
\hline Cell Radius $R$ & Normalized to 1 \\
\hline Noise Power Spectral Density $N_{0}$ & $-174 \mathrm{dBm} / \mathrm{Hz}$ \\
\hline Bandwidth $W$ & $10 \mathrm{MHz}$ \\
\hline Max. No. of Cooperative Set Degree $N_{c}$ & 6 \\
\hline No. of BS Antenna & 2 \\
\hline No. of Sectors $N_{s}$ & 3 \\
\hline SINR threshold $\gamma_{t h}$ & $10 \mathrm{~dB}$ \\
\hline
\end{tabular}

Table 4.2: System Parameters for the Power Consumption Model

\begin{tabular}{|l|l|}
\hline Parameter & Value Used \\
\hline Transmit Power $P_{t x}$ & 20 Watts \\
\hline$P_{\text {Misc }}$ & 0 \\
\hline Efficiency of DC/DC Converter $\mu_{d c / d c}$ & 0.94 \\
\hline Efficiency of Rectifier $\mu_{\text {rect }}$ & 0.952 \\
\hline$\beta_{0}$ & 0 \\
\hline$\beta_{1}$ & 0.65 \\
\hline Energy Efficiency Rating of Air Cond. $\eta$ & 13 \\
\hline Antenna system efficiency $\omega$ & 0.70 \\
\hline No. of Transmit Antennas per Sector $\mathrm{M}$ & 2 \\
\hline Max. No. of Cooperative Set Degree $N_{c}$ & 6 \\
\hline No. of Sectors $N_{s}$ & 3 \\
\hline Pilot Density per Channel p & $8 / 168$ \\
\hline Quantization bits per complex pilot $\mathrm{q}$ & 8 \\
\hline Symbol Period $T_{s}$ & $66.7 \mu \mathrm{sec}$ \\
\hline
\end{tabular}




\subsection{SINR at Worst Case Locations}

In order to meet the level of SINR at the worst case locations in conventional base station operation, the difference in SINR at a worst case location in conventional and in CoMP operation represents the increase in transmit power needed to meet that level of SINR.

Fig. 4.1 shows SINR for the worst case locations when there is cooperation among base stations and in case of conventional operation of base stations. If the quality of service requirements e.g the SINR threshold for communication is met at the worst case locations then at all other locations there shall not be any problem in meeting the desired minimum QoS.

In Fig. 4.1, SINR value at the worst case location in Mode No.7 is $11.4662 \mathrm{~dB}$ while SINR at the same worst case location in case of conventional operation of base station, is 18.0110 dB. An increase in transmit power by $6.5448 \mathrm{~dB}$ is needed in case of CoMP operation of base stations to meet exactly the same QoS as in case of conventional operation of base stations due to an increase in BS-UE distance. The increase in transmit power shall also cause an increase in interference elsewhere in the network. While in case of Modes No.2 and 3, SINR values at the worst case locations of these CoMP modes is greater than the SINR value at these locations in case of conventional base station operation thanks to base stations cooperation.

Let the SINR threshold $\left(\gamma_{t h}\right)$ be $10 \mathrm{~dB}$. Then in Fig. 4.1 it is evident that in Modes No.1, $2,3,4,5$ and 7 the required SINR threshold for communication is met.

In the remaining Modes of CoMP operation of base stations, the SINR value is much less than the threshold. Therefore the feasible Modes of operations meeting the minimum SINR threshold are Mode No.1, 2, 3, 4, 5 and 7. In the remaining Modes of operation it shall not be possible to meet the desired quality of service. 


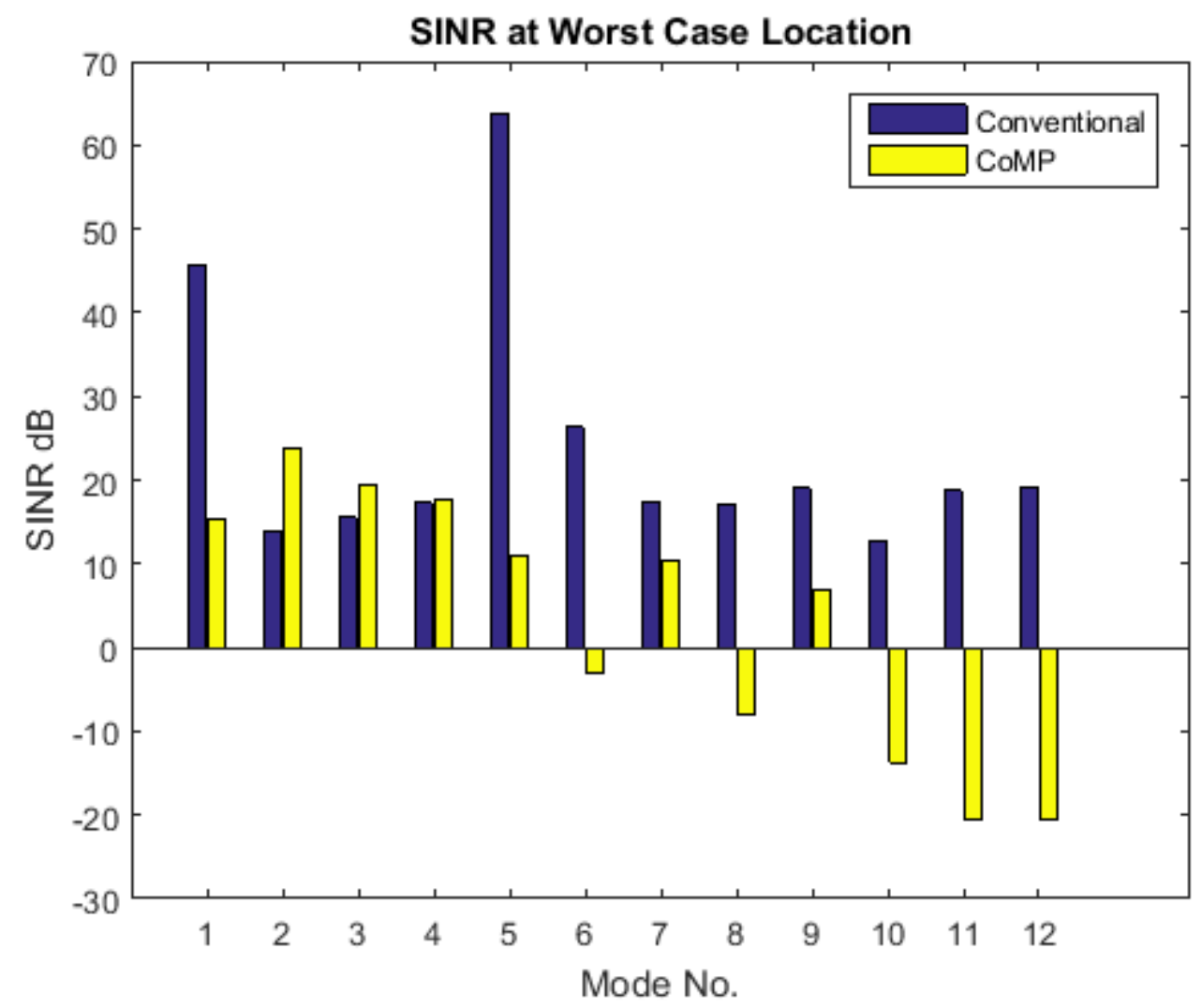

Figure 4.1: SINR at Worst Case Locations 


\subsection{Effect of $N_{c}$ on Power Consumption of Base Sta- tions Taking Part in CoMP Transmission}

As shown in Fig. 3.1 in Chapter 3 the total power required at BS site consists of the power required for backhauling, air conditioning and base station equipment which includes rectifier, DC/DC converter, SPU and RRU. The power required for alarm system, monitoring and ligting i.e $P_{\text {Misc }}$ has not been taken into consideration in this work.

Fig. 4.2 shows a comparison of power required for backhauling, air conditioning and BS hardware including rectifier and DC/DC converter. This graph is plotted for different values of $N_{c}$ i.e the number of BS taking part in CoMP transmission in the coverage area of the switched off BS i.e $C-0$.

When $N_{c}=1$ then it means that there is no cooperation among the BS's and only one BS is covering a particular area. As the number of BS's taking part in CoMP transmission increases from 2 to 6 , there is not any significant increase in backhauling power requirement. However there is tremendous increase in power required for BS subsystems, rectifier and DC/DC converter mainly due to increased processing of signals in SPU and increased power requirement of RRU Subsystem.

The increased processing in SPU and increased power required for RRU cause an increase in heat generation within the BS site and subsequently more energy is consumed by the air conditioning unit to remove that increased heat from the BS site.

\subsection{Comparision of BS Subsystems Power Requirement}

Fig. 4.3 shows the power needed for different subsystems of a base station. Some of the power input to rectifier and DC/DC converter is converted into heat and the rest is used for the operation of SPU and RRU Subsystems. 


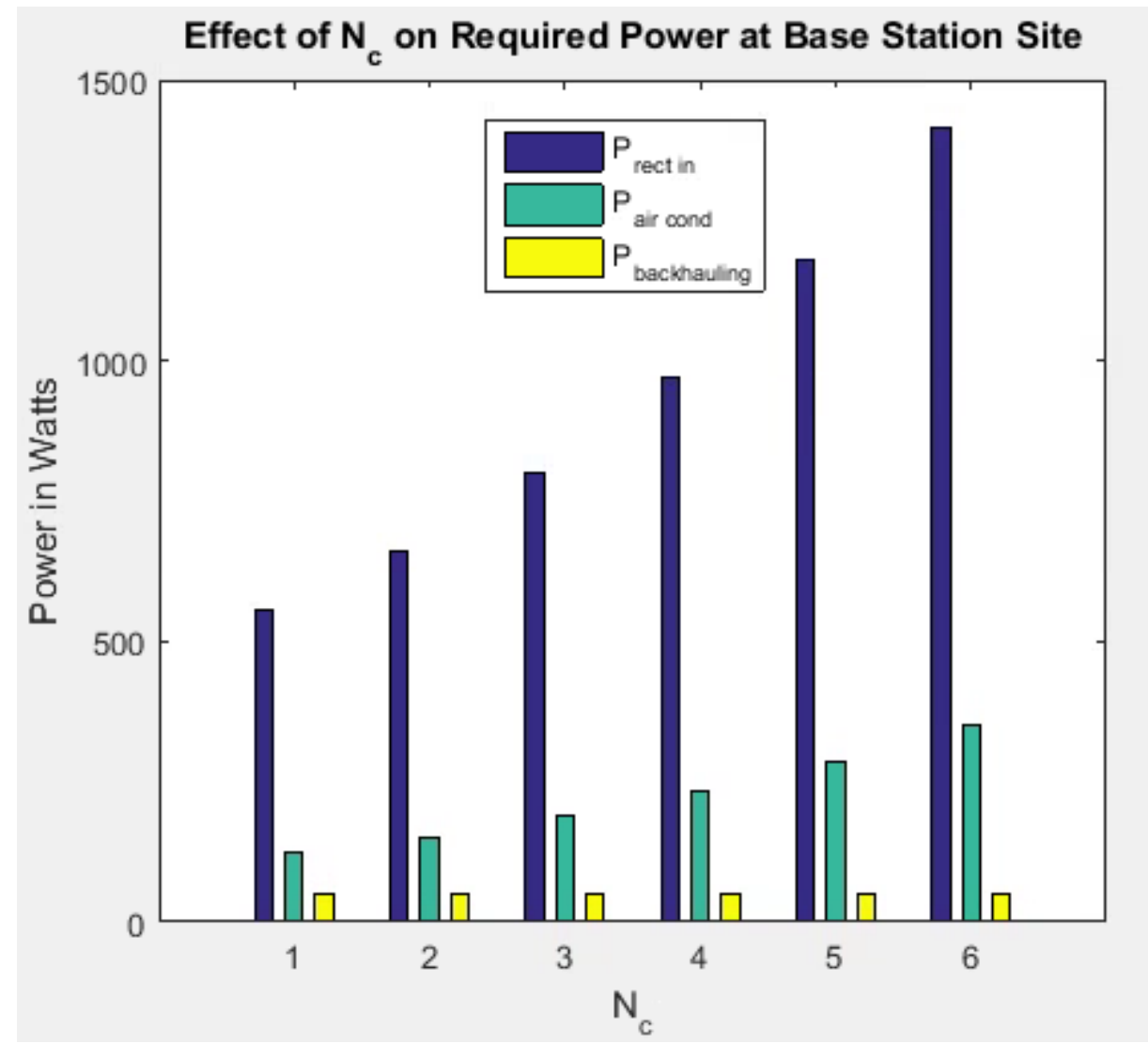

Figure 4.2: Change in Required Power with Number of BS Taking Part in CoMP Transmission 
The power consumption of rectifier and DC/DC converter are calculated using following equations.

$$
\begin{gathered}
P_{\text {rect-cons }}=\frac{P_{\text {rect-out }}\left(1-\mu_{\text {rect }}\right)}{\mu_{\text {rect }},} . \\
P_{d c / d c-c o n s}=\frac{P_{d c-d c_{\text {out }}}\left(1-\mu_{d c-d c}\right)}{\mu_{d c-d c}},
\end{gathered}
$$

whereas $P_{\text {rect-out }}=\mu_{\text {rect }} . P_{\text {rect-in }}$ and $P_{d c-d c_{o u t}}=\frac{P_{s p-C o M P}}{1-\beta_{1}}$.

The results of Fig. 4.3 show that there is significant increase in power requirement of SPU with increasing the value of $N_{c}$.

Based on these results, in order to reduce overall power requirements and to reduce energy consumption of BS site, any future reserach effort needes to be directed towards SPU. Any significant decrease in SPU energy requirements shall greatly improve the energy efficiency of BS site and the whole cellular network.

\subsection{Energy Efficiency Ratio of Conventional and CoMP Feasible Switching Modes}

Table 4.3 shows that capacity of user in the switched off cell area increases with increasing the value of $N_{c}$. However the increase in energy consumption with increasing value of $N_{c}$ is far greater than the increase in capacity with $N_{c}$.

Table 4.3: User Capacity of Feasible Switching Modes

\begin{tabular}{|l|l|}
\hline Mode No. & Capacity(bps) $\times 10^{8}$ \\
\hline 1 & 4.9148 \\
\hline 2 & 4.8885 \\
\hline 3 & 4.8563 \\
\hline 4 & 4.8563 \\
\hline 5 & 4.8563 \\
\hline 7 & 4.8148 \\
\hline
\end{tabular}




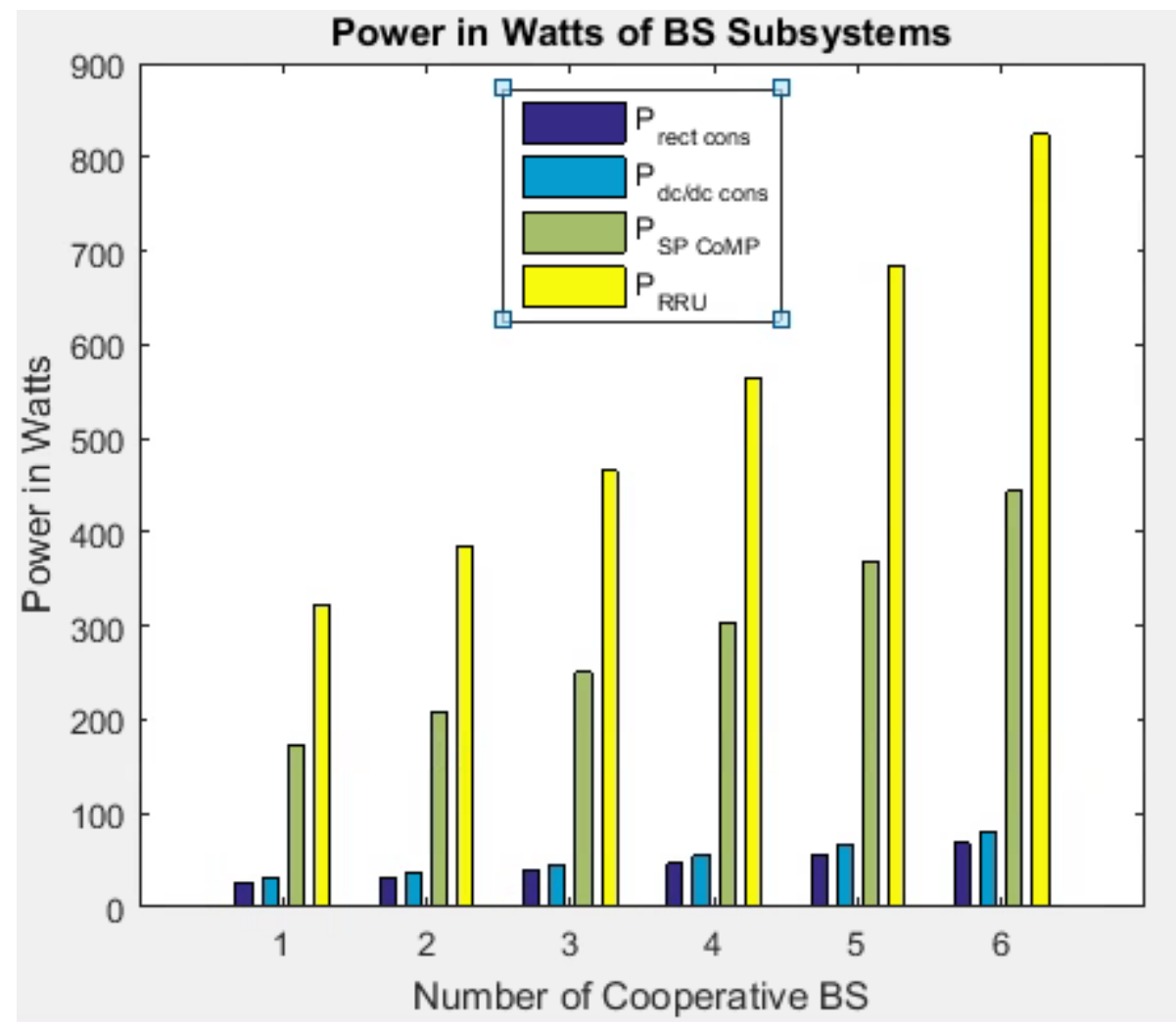

Figure 4.3: Subsystems Power in Watts

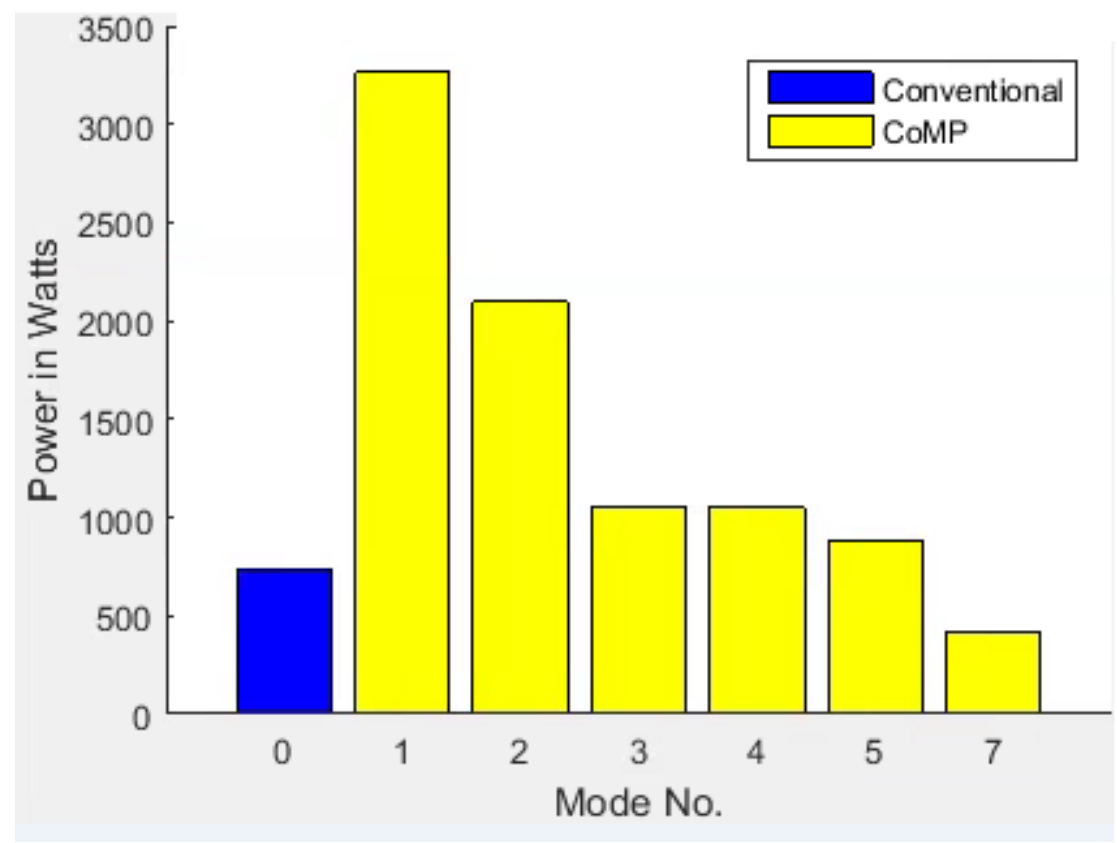

Figure 4.4: Power Consumption in Different Switching Modes to Provide Service in C-0 
In Fig. 4.4 the power consumption in conventional operation of BS and in feasible CoMP modes is shown. Only in Mode 7 the power consumption to provide service in the switched off cell coverage area is less than the conventional method.

Similarly EER in bits per Joule is better in conventional operation of BS compared to CoMP operation of BS's except in Mode 7. Switching Mode 7 is more energy efficient than the conventional operation of BS thanks to a lower value of $N_{c}$ i.e 3 . However increasing the value of $N_{c}$ beyond 3 causes EER to decrease for example in Modes 1, 2, 3, 4, 5 .

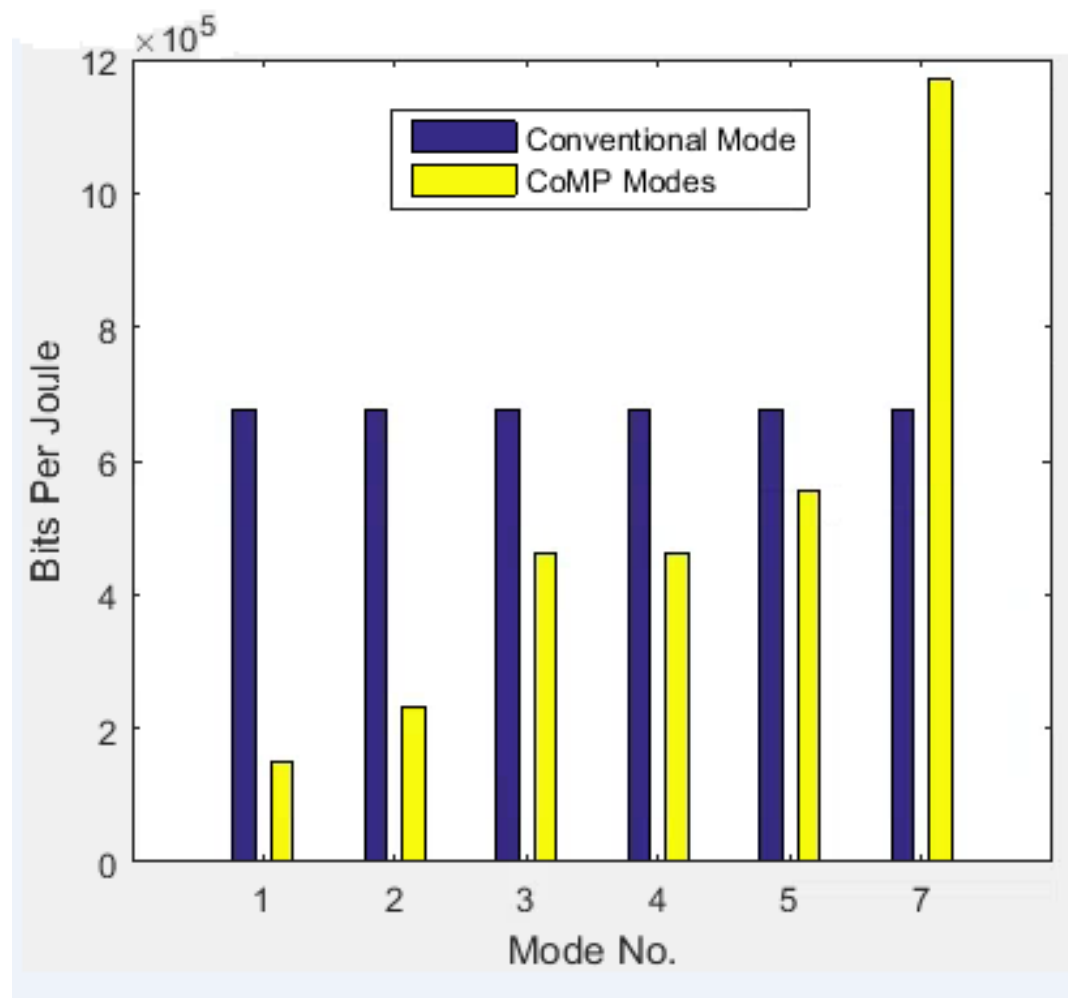

Figure 4.5: Bits per Joule of Feasible Modes 


\subsection{Analysis of Mode No. 6 and Mode No. 7}

Three base stations are taking part in CoMP transmission in Mode No. 6 and in Mode No. 7. In Mode No. 6 base stations identified by cells $C-1, C-3$ and $C-5$ are taking part in CoMP transmission in the coverage area of the switched off cell $C-0$. The interfering cells in this mode are $C-2, C-4, C-6, C-1^{\prime}, C-2^{\prime}, C-3^{\prime}, C-4^{\prime}, C-5^{\prime}, C-6^{\prime}, C-7^{\prime}, C-$ $8^{\prime}, C-9^{\prime}, C-10^{\prime}, C-11^{\prime}$ and $C-12^{\prime}$. In Mode No. 7 three base stations identified by cells $C-1, C-4$ and $C-6$ are taking part in CoMP transmission in the switched off cell area $C-0$. The number of interfering cells in this mode are $C-5, C-1^{\prime}, C-9^{\prime}, C-10^{\prime}, C-11^{\prime}$ and $C-12^{\prime}$. In both modes the number of CoMP base stations is three however the number of interfering cells is different effecting the SINR values at the worst case locations in each mode differently.

In Mode No. 6 two sectors of each CoMP base station i.e sectors 1 and 2 of BS $B_{1}$, sectors 2 and 3 of BS $B_{3}$ and sectors 1 and 3 of BS $B_{5}$ are taking part in CoMP transmission. However in Mode No. 7 two sectors of BS $B_{1}$ i.e sector 1 and 2, one sector of $\mathrm{BS} B S_{4}$ i.e sector 3 and one sector of $\mathrm{BS} B_{6}$ i.e sector 1 is taking part in CoMP transmission in the switched off coverage area $C-0$ thereby resulting a much lower power consumption in Mode No. 7 as compared to Mode No. 6. The power required in Mode No. 6 to cover the switched off cell $C-0$ coverage area is 617.32 Watts which is 205.72 Watts greater than in Mode No. 7. Therefore Mode No. 7 is preferred over Mode No. 6 .

\subsection{Application in a Cellular Network}

Low traffic phenomenon can be different in night than in day time. Normally an extremely low traffic is observed in all BS's during night times i.e after midnight till early morning. However in day times within a particular geographical area some BS's may be experiencing 
low traffic (e.g residential areas) while others may be experiencing very high raffic or even congestion (e.g commercial areas and areas where offices are located).

Traffic reports of two different BS's of a telecom operator PTCL are shown in Table 4.5 and Table 4.6 from midnight till 7:00 am.

An application of the work of this thesis during off-peak traffic periods during night is shown in Fig. 4.6.

Table 4.4: Base Station (Khar Bajur-NTR1P2138) Traffic During Night Times

\begin{tabular}{|c|c|}
\hline Date and Time & No. of Calls \\
\hline 2012-12-15 00:00:00 & 9 \\
\hline 2012-12-15 00:00:00 & 5 \\
\hline $2012-12-15$ 00:00:00 & 25 \\
\hline 2012-12-15 01:00:00 & 28 \\
\hline 2012-12-15 01:00:00 & 1 \\
\hline 2012-12-15 01:00:00 & 20 \\
\hline $2012-12-15$ 02:00:00 & 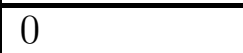 \\
\hline $2012-12-15$ 02:00:00 & 0 \\
\hline 2012-12-15 02:00:00 & 8 \\
\hline 2012-12-15 03:00:00 & 1 \\
\hline $2012-12-15$ 03:00:00 & 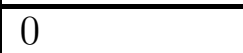 \\
\hline $2012-12-15$ 03:00:00 & 4 \\
\hline $2012-12-15$ 04:00:00 & $\overline{0}$ \\
\hline 2012-12-15 04:00:00 & 1 \\
\hline $2012-12-15$ 04:00:00 & 14 \\
\hline $2012-12-15$ 05:00:00 & 11 \\
\hline $2012-12-15$ 05:00:00 & 10 \\
\hline 2012-12-15 05:00:00 & 35 \\
\hline $2012-12-15$ 06:00:00 & 30 \\
\hline 2012-12-15 06:00:00 & 51 \\
\hline $2012-12-15$ 06:00:00 & 264 \\
\hline $2012-12-15$ 07:00:00 & 117 \\
\hline 2012-12-15 07:00:00 & 144 \\
\hline $2012-12-15$ 07:00:00 & 554 \\
\hline
\end{tabular}

Three types of cells are identified in Fig. 4.6. 


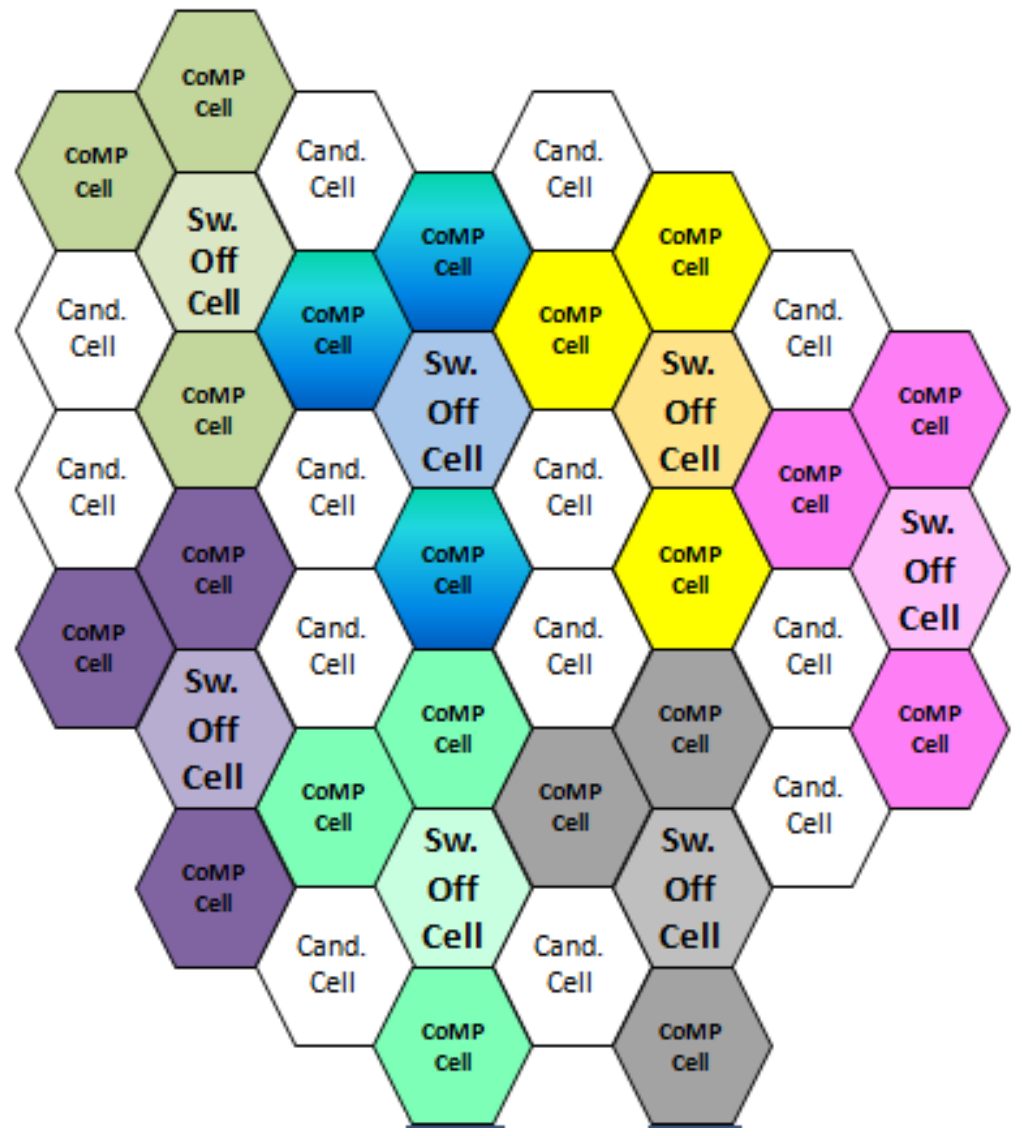

Figure 4.6: Extension of Mode No.7 in a Cellular Network 
Table 4.5: Base Station (Reshun-NTR1P4157) Traffic During Night Times

\begin{tabular}{|l|l|}
\hline Date and Time & No. of Calls \\
\hline 2012-12-15 00:00:00 & 18 \\
\hline 2012-12-15 00:00:00 & 38 \\
\hline 2012-12-15 00:00:00 & 0 \\
\hline $2012-12-15$ 01:00:00 & 0 \\
\hline $2012-12-15$ 01:00:00 & 4 \\
\hline $2012-12-15$ 01:00:00 & 0 \\
\hline $2012-12-15$ 02:00:00 & 3 \\
\hline $2012-12-15$ 02:00:00 & 4 \\
\hline $2012-12-15$ 02:00:00 & 0 \\
\hline $2012-12-15$ 03:00:00 & 3 \\
\hline $2012-12-15$ 03:00:00 & 3 \\
\hline $2012-12-15$ 03:00:00 & 0 \\
\hline $2012-12-15$ 04:00:00 & 3 \\
\hline $2012-12-15$ 04:00:00 & 14 \\
\hline $2012-12-15$ 04:00:00 & 0 \\
\hline $2012-12-15$ 05:00:00 & 12 \\
\hline $2012-12-15$ 05:00:00 & 35 \\
\hline $2012-12-15$ 05:00:00 & 0 \\
\hline $2012-12-15$ 06:00:00 & 70 \\
\hline $2012-12-15$ 06:00:00 & 115 \\
\hline $2012-12-15$ 06:00:00 & 0 \\
\hline $2012-12-15$ 07:00:00 & 162 \\
\hline $2012-12-15$ 07:00:00 & 203 \\
\hline $2012-12-15$ 07:00:00 & 0 \\
\hline
\end{tabular}

Switched Off Cells: The cells that can be switched off due to extremely low traffic level provided a set of neighbouring cells are available to provide service in the switched off cell coverage area in Mode No. 7.

CoMP Cells: The cells providing service in the switched off cell coverage area using CoMP transmission and working in Mode No. 7.

Candidate Cells: Which are experiencing low traffic and can take part in CoMP transmission in the switched off cell area if a need arises i.e if any of the CoMP cell gets out 
of cooperation due to increase in its own traffic or due any other interruption such as BS hardware, software problems, or transmission media failure. The candidate cell can also operate to cover another switched off cell area if it has enough spare capacity to serve two switched off cells at a time.

To further apply the results of this work in the cellular network, consider a geographical area covered by 42 cells as shown in Fig 4.6. The area of a regular hexagon is $\frac{3 \sqrt{3}}{2} R^{2}$, and the total area of the cells in Fig. 4.6 is calculated as $42(2.59) R^{2}$.

During off-peak periods in the night, all BS's experience extremely low traffic. The number of BS's that can be switched off are 7. In Fig. 4.4 the amount of required power to provide service in a cell in Mode 0, i.e in conventional manner is 729.30 Watts; while providing service in the same cell by neighbouring BS's using CoMP transmission requires 411.60 Watts, saving 317.71 Watts power in Mode No. 7. The saving in energy in Joules is given as

$$
E_{\text {saved }}=P_{\text {saved }} \cdot \text { time }
$$

whereas $E_{\text {saved }}$ is the amount of energy saved in Joules, $P_{\text {saved }}$ is the power saved in Watts and time is the time interval in seconds during off-peak traffic hours.

Considering the time from 00:00 hrs in the night till 06:00 a.m to be the off-peak period (seven BS's remain switched off for six hours a day) which is also evident from Fig. 1.2 and Fig. 3.1 the number of units of electricity saved in one year for the cellular network shown in Fig. 4.6 can be calculated as $\frac{(317.71) \cdot 67.765}{1000}=4803.77 \mathrm{kWh}$.

In Canada the $4803.77 \mathrm{kWh}$ units equivalent Carbon Dioxide $\left(\mathrm{CO}_{2} e\right)$ is 4.04 tons for Alberta, while for Ontario it is 0.62 tons using Carbon emission calculator [29]. The difference in $\mathrm{CO}_{2} \mathrm{e}$ between the two provinces for the same number of electricity units is due to the difference in energy mix. In ontario only $28 \%$ of power generation is from oil and gas while in Alberta 90\% of power is generated using oil and gas [30], [31]. 
Seven cells have been switched off in Fig. 4.6 and the amount of power saved per cell switched off is 729.3-411.6=317.7 Watts as is evident from Fig. 4.4. The total amount of power saved in the network layout of Fig. 4.6 is $(7)(317.7)=2224$ Watts. The power required to operate 42 cells in conventional manner is $(42)(729.3)=30630$ Watts. The saving is $7.26 \%$.

Considering the number of BS's which have reached 4 million figure worldwide by 2012 [2] a large amount of power can be saved by properly applying CoMP technique.

In real networks consisting of pico, micro and macro cells, the pico cells can be turned off in stage 1 and the area to be serviced by the micro cells. Then in stage 2 some of the micro cells can also be turned off to save further energy. 


\section{Chapter 5}

\section{Conclusion}

\subsection{Summary and Contributions}

In this thesis an effort has been made to investigate the usage of CoMP in order to provide service in an area whose BS is switched off during off-peak traffic periods for saving its operational energy. The switched off coverage area is then served using CoMP transmission by neighbouring base stations.

The QoS should not deteriorate in the switched off cell coverage area when the neighbouring base stations are serving that particular switched off cell area, besides ensuring overall energy saving.

The usage of CoMP causes an increase in the energy consumption of the neighbouring base stations. It has been observed that in some switching modes the SINR value at worst case locations during CoMP transmission in switched off areas has deteriorated compared to conventional operation. The switching modes that do not satisfy the required SINR threshold at worst case locations are considered as non feasible.

Ensuring exactly similar QoS in both cases is difficult however the worst case locations within each of the twelve switching modes are used as reference, and assuming that if the 
SINR threshold $\left(\gamma_{t h}\right)$ is met at these locations then it can be said that at other locations which are closer to the base stations, the signal quality shall be much more better than the minimum required threshold.

The mobile user is free to roam within a cell and the PDF of random distance within a cell and within an adjoining cell is used to find out the expected value of SNR for every mode. The capacity based on the expected value of SNR is then calculated for every mode. It has been found that the capacity of user has increased with the increase in number of BS taking part in CoMP transmission. The power consumption also increases with value of $N_{c}$ and this increase is much greater than the increase in capacity with $N_{c}$, thereby causing a much lower value of EER in bit/Joule for modes in which $N_{c}$ value is greater.

A power consumption model for BS's taking part in CoMP transmission to provide service in a particular switched off BS area is developed. This model provides an insight into the power consumption of various subsystems i.e SPU, RRU, rectifier, DC/DC Conveter and air conditioner at the BS site. The effect of $N_{c}$ i.e the number of BS taking part in CoMP transmission is evaluated, indicating that increasing the value of $N_{c}$ shall decrease EER.

Since BS switching off is considered at extremely low traffic levels, therefore the effect of traffic on power consumption has not been taken into account.

EER is found as bit/Watts-sec=bits/Joule. The observations of the work revealed that aggresive usage of CoMP decreases EER. There is an increase in capacity with increasing the number of BS's taking part in CoMP transmission but the associated increase in energy consumption is much higher. The maximum number of BS taking part in CoMP tranmission that can ensure energy saving is found to be three.

In all other switching modes (e.g using a value of $N_{c}$ less than three) either the QoS cannot be ensured or the EER is worse (e.g using a value of $N_{c}$ greater than three) compared to that by conventional operation.

The work is then applied to a network of 42 BS's and considering the off-peak period 
after midnight, the amount of power that can be saved within a small network of just 42 cells is calculated. The amount of Carbon Dioxide equivalent $\left(\mathrm{CO}_{2} e\right)$ is then calculated for the provinces of Alberta and Ontario with different energy mix.

\subsection{Future Research Directions}

Results of the power consumption model show that RRU and SPU require most of the BS power therefore further research is needed to reduce power requirements of these subsystems. Any reduction in energy requirements of these two subsystems shall greatly improve EER of cellular networks in case CoMP transmission is used to provide coverage in the switched off cell area.

In this work, the network model consists of cells of the same size; however in real networks, cells are of different sizes and further research can be carried out using a heterogeneous network consisting of femto, pico, micro and macro cells.

In this work the transmit power of CoMP BS's has not been increased, the effect of increasing the transmit power of the neighbouring CoMP BS's on the capacity and EER of switched off cell and on the capacity of other cells not taking part in CoMP transmission is yet another area to be further researched.

In this work only downlink has been considered, however a more realistic approach can be to consider both uplink and downlink.

The effect of traffic on the BS power consumption has been ignored in this work, in any future work its effect can be taken into account. 


\section{Appendix A}

\section{Abbreviation List}

$\begin{array}{ll}\text { BS } & \text { Base Station } \\ \text { BTU } & \text { British Thermal Unit } \\ \text { CoMP } & \text { Coordinated Multipoint } \\ \text { CBF } & \text { Cooperative Beam Forming } \\ \text { CSI } & \text { Channel State Information } \\ \text { ECR } & \text { Energy Consumption Ratio } \\ \text { EER } & \text { Energy Efficiency Ratio } \\ \text { FFR } & \text { Flexible Frequency Reuse } \\ \text { HPA } & \text { High Power Amplifier } \\ \text { LNA } & \text { Low Noise Amplifier } \\ \text { LTE-A } & \text { Long Term Evolution-Advanced } \\ \text { MIMO } & \text { Multiple-Input Multiple-Output } \\ \text { MISO } & \text { Multiple-Input Single-Output } \\ \text { PDF } & \text { Probability Density Function } \\ \text { RRU } & \text { Radio Resource Unit } \\ \text { PTCL } & \text { Pakistan Telecommunication Company Limited } \\ \text { SPU } & \text { Signal Processing Unit } \\ \text { SINR } & \text { Signal to Interference plus Noise Ratio } \\ \text { SNR } & \text { Signal to Noise Ratio } \\ \text { UE } & \text { User Equipment }\end{array}$




\section{Appendix B}

\section{Integration}

Let

$$
\begin{gathered}
I_{1}=\int x^{-3} \cos ^{-1} \frac{\sqrt{3}}{2 x} d x \\
I_{2}=\int x^{-3} \frac{\pi}{6} d x \\
I_{3}=\int x^{-3} \sin ^{-1} \frac{3-\sqrt{x^{2}-3}}{2 x} d x
\end{gathered}
$$

First considering $I_{1}$

Let $x^{-1}=t$

$$
\begin{gathered}
x^{-2} d x=-d t \\
I_{1}=-\int t \cos ^{-1} \frac{\sqrt{3}}{2} d t=-\left\{\frac{t^{2}}{2} \cos ^{-1} \frac{\sqrt{3}}{2} t+\int \frac{t^{2}}{2} \frac{\sqrt{3}}{2} \frac{1}{\sqrt{1-\left(\sqrt{\frac{3}{2}} t\right)^{2}}} d t\right\}
\end{gathered}
$$

According to [32]

$$
\begin{aligned}
& \int t^{2} \frac{}{\sqrt{a^{2}-t^{2}}} d t=\frac{a^{2}}{2} \sin ^{-1} \frac{t}{a}-\frac{1}{2} t \sqrt{a^{2}-t^{2}} \\
& I_{1}=-\left\{\frac{t^{2}}{2} \cos ^{-1} \frac{\sqrt{3}}{2} t+\frac{1}{2} \frac{\sqrt{3}}{2} \int t^{2} \frac{}{\frac{\sqrt{3}}{2} \sqrt{\frac{2^{2}}{\sqrt{3}^{2}}-t^{2}}} d t\right\}
\end{aligned}
$$




$$
I_{1}=-\frac{t^{2}}{2} \cos ^{-1} \frac{\sqrt{3}}{2} t-\frac{1}{3} \sin ^{-1} \frac{\sqrt{3}}{2} t+\frac{1}{4} t \sqrt{\frac{4}{3}-t^{2}}
$$

Next considering integration of $I_{2}$

$$
\begin{gathered}
I_{2}=\int t^{-3} \frac{\pi}{6} d t \\
I_{2}=-\frac{\pi}{12} t^{-2}
\end{gathered}
$$

Next considering integration of $I_{3}$

$$
I_{3}=\int x^{-3} \sin ^{-1} \frac{3-\sqrt{x^{2}-3}}{2 x} d x
$$

Let

$$
\begin{aligned}
& x=\sqrt{3} \sec \theta \\
& d x=\sqrt{3} \sec \theta \tan \theta d \theta \\
& I_{3}=\int(\sqrt{3} \sec \theta)^{-3} \sin ^{-1} \frac{3-\sqrt{(\sqrt{3} \sec \theta)^{2}-(\sqrt{3})^{2}}}{2 \sqrt{3} \sec \theta} \sqrt{3} \sec \theta \tan \theta d \theta \\
& I_{3}=\int \frac{\tan \theta}{3(\sec \theta)^{2}} \sin ^{-1}\left(\frac{3-\sqrt{3} \tan \theta}{2 \sqrt{3} \sec \theta}\right) d \theta \\
& I_{3}=\int \frac{\sin \theta \cos \theta}{3} \sin ^{-1}\left(\frac{\sqrt{3}}{2} \cos \theta-\frac{\sin \theta}{2}\right) d \theta \\
& I_{3}=\int \frac{\sin 2 \theta}{6} \sin ^{-1}\left(\frac{\sqrt{3}}{2} \cos \theta-\frac{\sin \theta}{2}\right) d \theta \\
& I_{3}=\frac{1}{6}\left\{-\frac{\cos 2 \theta}{2} \sin ^{-1}\left(\frac{\sqrt{3}}{2} \cos \theta-\frac{\sin \theta}{2}\right)+\frac{1}{2} \int\left(\cos 2 \theta \frac{d}{d \theta} \sin ^{-1}\left(\frac{\sqrt{3}}{2} \cos \theta-\frac{\sin \theta}{2}\right)\right) d \theta\right\} \\
& I_{3}=\frac{1}{6}\left\{-\frac{\cos 2 \theta}{2} \sin ^{-1}\left(\frac{\sqrt{3}}{2} \cos \theta-\frac{\sin \theta}{2}\right)+\frac{1}{2} \int \cos 2 \theta \frac{1}{\sqrt{1-\left(\frac{\sqrt{3}}{2} \cos \theta-\frac{1}{2} \sin \theta\right)^{2}}} \frac{d}{d \theta}\left(\frac{\sqrt{3}}{2} \cos \theta-\frac{1}{2} \sin \theta\right) d \theta\right\} \\
& I_{3}=\frac{1}{6}\left\{-\frac{\cos 2 \theta}{2} \sin ^{-1}\left(\frac{\sqrt{3}}{2} \cos \theta-\frac{\sin \theta}{2}\right)+\frac{1}{2} \int \cos 2 \theta \frac{1}{\sqrt{1-\frac{1}{4}(\sqrt{3} \cos \theta-\sin \theta)^{2}}}\left(\frac{-\sqrt{3}}{2} \sin \theta-\frac{1}{2} \cos \theta\right) d \theta\right\}
\end{aligned}
$$




$$
\begin{aligned}
& I_{3}=\frac{1}{6}\left\{-\frac{\cos 2 \theta}{2} \sin ^{-1}\left(\frac{\sqrt{3}}{2} \cos \theta-\frac{\sin \theta}{2}\right)+\frac{1}{2} \int \cos 2 \theta \frac{\left(\frac{-\sqrt{3}}{2} \sin \theta-\frac{1}{2} \cos \theta\right)}{\sqrt{1-\frac{1}{4}\left(3 \cos ^{2} \theta+\sin ^{2} \theta-2 \sqrt{3} \cos \theta \sin \theta\right)}} d \theta\right\} \\
& I_{3}=\frac{1}{6}\left\{-\frac{\cos 2 \theta}{2} \sin ^{-1}\left(\frac{\sqrt{3}}{2} \cos \theta-\frac{\sin \theta}{2}\right)+\frac{1}{2} \int \cos 2 \theta \frac{\left(\frac{-\sqrt{3}}{2} \sin \theta-\frac{1}{2} \cos \theta\right)}{\sqrt{\frac{1}{4}\left(4-\left(3-3 \sin ^{2} \theta+\sin ^{2} \theta-2 \sqrt{3} \cos \theta \sin \theta\right)\right)}} d \theta\right\} \\
& I_{3}=\frac{1}{6}\left\{-\frac{\cos 2 \theta}{2} \sin ^{-1}\left(\frac{\sqrt{3}}{2} \cos \theta-\frac{\sin \theta}{2}\right)+\frac{1}{2} \int \cos 2 \theta \frac{\left(\frac{-\sqrt{3}}{2} \sin \theta-\frac{1}{2} \cos \theta\right)}{\sqrt{\frac{1}{4}\left(1+2 \sin ^{2} \theta+2 \sqrt{3} \cos \theta \sin \theta\right)}} d \theta\right\} \\
& I_{3}=\frac{1}{6}\left\{-\frac{\cos 2 \theta}{2} \sin ^{-1}\left(\frac{\sqrt{3}}{2} \cos \theta-\frac{\sin \theta}{2}\right)+\frac{1}{2} \int \cos 2 \theta \frac{\left(\frac{-\sqrt{3}}{2} \sin \theta-\frac{1}{2} \cos \theta\right)}{\sqrt{\frac{1}{4}\left(\sin ^{2} \theta+\cos ^{2} \theta+2 \sin ^{2} \theta+2 \sqrt{3} \cos \theta \sin \theta\right)}} d \theta\right\} \\
& I_{3}=\frac{1}{6}\left\{-\frac{\cos 2 \theta}{2} \sin ^{-1}\left(\frac{\sqrt{3}}{2} \cos \theta-\frac{\sin \theta}{2}\right)+\frac{1}{2} \int \cos 2 \theta \frac{\left(\frac{-\sqrt{3}}{2} \sin \theta-\frac{1}{2} \cos \theta\right)}{\sqrt{\frac{1}{4}\left(3 \sin ^{2} \theta+\cos ^{2} \theta+2 \sqrt{3} \cos \theta \sin \theta\right)}} d \theta\right\} \\
& I_{3}=\frac{1}{6}\left\{-\frac{\cos 2 \theta}{2} \sin ^{-1}\left(\frac{\sqrt{3}}{2} \cos \theta-\frac{\sin \theta}{2}\right)+\frac{1}{2} \int \cos 2 \theta \frac{\left(\frac{-\sqrt{3}}{2} \sin \theta-\frac{1}{2} \cos \theta\right)}{\sqrt{\frac{1}{4}(\sqrt{3} \sin \theta+\cos \theta)^{2}}} d \theta\right\} \\
& I_{3}=\frac{1}{6}\left\{-\frac{\cos 2 \theta}{2} \sin ^{-1}\left(\frac{\sqrt{3}}{2} \cos \theta-\frac{\sin \theta}{2}\right)-\frac{1}{4} \int \cos 2 \theta d \theta\right. \\
& I_{3}=\frac{1}{6}\left\{-\frac{\cos 2 \theta}{2} \sin ^{-1}\left(\frac{\sqrt{3}}{2} \cos \theta-\frac{\sin \theta}{2}\right)-\frac{1}{8} \sin 2 \theta\right\}
\end{aligned}
$$




\section{Bibliography}

[1] J. Wu, Y. Zhang, M. Zukerman, "Energy-efficient base-stations sleep-mode techniques in green cellular networks: A survey", IEEE Communications Surveys and Tutorials, vol. 17, no. 2, pp. 803-826, Feb. 2015.

[2] C. H. Tang, C. E. Wu, C. W. Lin and C. Y. Liao, "Network energy efficiency for deployment architectures with base station site model", 1st IEEE International Conference on Communications in China Workshops, Beijing, pp. 85-90, Aug. 2012.

[3] J. J. Q. Yu and V. O. K. Li, "Base station switching problem for green cellular networks with Social Spider Algorithm ", IEEE Congress on Evolutionary Computation, Beijing, pp. 2338-2344, July 2014.

[4] Z. Niu, "TANGO:Traffic-Aware network planning and green operation", IEEE Wireless Communications, vol. 18, no. 5, pp. 25-29, Oct. 2011.

[5] Z. Hasan, H. Boostanimehr, V. K. Bhargava "Green cellular networks: A survey, some research issues and challenges", IEEE Communications Surveys and Tutorials, vol. 13, no. 4, pp. 524-540, Nov. 2011.

[6] J. Lorincz, T. Garma and G. Petrovic, "Measurements and modelling of base station power consumption under real traffic loads ", Sensors, vol. 12, no. 4, pp. 4281-4310, March 2012. 
[7] M. A. Marsan and M. Meo, "Energy efficient management of two cellular access networks", ACM SIGMETRICS Performance Evaluation Review, vol. 37, no. 4, pp. 69-73, March 2010.

[8] Z. Niu, Y. Wu, J.Gong and Z.Yang, "Cell zooming for cost-efficient green cellular networks", IEEE Communications Magazine, vol. 48, no. 11, pp. 74-79, Nov. 2010.

[9] T. Han and N. Ansari, "On greening cellular networks via multicell cooperation", IEEE Wireless Communications Magazine, vol. 20, no. 1, pp. 82-89, Feb. 2013.

[10] L. M. Correia, D. Zeller, O. Blume, D. Ferling, "Challenges and enabling technologies for energy aware mobile radio networks", IEEE Communications Magazine, vol. 48, no. 11, pp. 66-72, Nov. 2010.

[11] S. Zoican, "The role of digital signal processors for 3G mobile communication systems", ACTA Tech. Napoc., vol. 49, pp. 49-56, 2008.

[12] G. Micallef, P. Mogensen, H. O. Scheck, J. Louhi, "Reversing the energy trend in mobile networks: equipment replacement for increased capacity at a fraction of the energy", IEEE vehicular technology conference (VTC Fall), San Francisco, pp. 1-5, Sep. 2011.

[13] E. Oh, K. Son, B. Krishnamachari, "Dynamic base station switching-on/off strategies for green cellular networks", IEEE Transactions on Wireless Communications, vol. 12, no. 5, pp. 2126-2136, May 2013.

[14] A. Davydov, G. Morozov, I. Bolotin, A. Papathanassiou, "Evaluation of joint transmission CoMP in C-RAN based LTE-A HetNets with large coordination areas", IEEE Globecom Workshops, Atlanta, pp. 801 - 806, Dec. 2013.

[15] X. Wang, P. Krishnamurthy and D. Tipper, "Cell sleeping for energy efficiency in cellular 
networks: is it viable?", IEEE Wireless Communications and Networking Conference, Shanghai, pp. 2509-2514, April 2012.

[16] R1-050507, "Soft frequency reuse scheme for UTRAN LTE", Huawei 3GPP TSG RAN WG1 meeting no. 41, Athens, Greece, May 2005.

[17] R. Irmer et al., "Coordinated multipoint: concepts, performance, and field trial results", IEEE Communications Magazine, vol. 49, no. 2, pp. 102-111, Feb. 2011,

[18] D. B. Cheikh et al., "Analytical joint processing multi-point cooperation performance in Rayleigh fading", IEEE Wireless Communication Letters, vol. 1, no. 4, pp. 272-275, April 2012.

[19] F. Han, Z. Safar and K. J. R. Liu, "Energy-efficient base station cooperative operation with guaranteed QoS", IEEE Transactions on Communications, vol. 61, no. 8, pp. 3505-3516, Aug. 2013.

[20] X. Ge et al., "Capacity analysis of a multi-cell multi-antenna cooperative cellular network with co-channel interference", IEEE Transactions on Wireless Communications, vol. 10, no. 10, pp. 3298-3309, Oct. 2011.

[21] Y. Zhuang, Y. Luo, L. Cai, J. Pan, "A geometric probability model for capacity analysis and interference estimation in wireless mobile cellular systems", IEEE Global Telecommunication Conference-GLOBECOM 2011, pp. 1-6, Dec. 2011.

[22] O. Apilo, M. Lasanen, S. Boumard, A. Mammela, "The distribution of link distances in distributed multiple-input multiple-output cellular systems", IEEE Conference on Vehicular Technology (VTC Spring-2013), pp. 1-5, June 2013.

[23] I. Humar et al., "Rethinking energy efficiency models of cellular networks with embodied Energy", IEEE Networks, vol. 25, no. 2, pp. 40-49, March-April 2011. 
[24] B. H. Jung, H. Leem and D. K. Sung, "Modeling of power consumption for macro-, micro-, and RRH-based base station architectures", 79th IEEE Vehicular Technology Conference (VTC Spring), pp. 1-5, May 2014.

[25] G. Auer et al., "How much energy is needed to run a wireless network?", IEEE Wireless Communications, vol. 18, no. 5, pp. 40-49, Oct. 2011.

[26] C.-H. Tang et al., "Network energy efficiency for deployment architectures with base station site model", 1st IEEE International Conference on Communications in China Workshops(ICCC), pp. 85-90, Aug. 2012.

[27] H. Holtkamp et al., "Minimizing base station power consumption ", IEEE Journal on Selected Areas in Communications, vol. 32, no. 2, pp. 297-306, Jan. 2014.

[28] G. Cili, H. Yanikomeroglu, F. R. Yu, "Cell switch off technique combined with coordinated multi-point transmission for energy efficiency in beyond-LTE cellular networks", IEEE International Conference on Communication, Ottawa, pp. 5931-5935, June 2012.

[29] http://www.carbonzero.ca/calculate, date accessed: March 012016.

[30] http://www.ieso.ca/Pages/Power-Data/Supply.aspx, date accessed: March 012016.

[31] http://www.energy.alberta.ca/electricity/682.asp, date accessed: date accessed: March 012016.

[32] R. L. Finney and M. D. Weir, Thomas' Calculus, 10th edition, Addison-Wesley, July 2000. 